\title{
A WIDE-ANGLE TAIL RADIO GALAXY IN THE COSMOS FIELD: EVIDENCE FOR CLUSTER FORMATION ${ }^{1}$
}

\author{
V. Smolčić, ${ }^{2,3}$ E. Schinnerer, ${ }^{2}$ A. Finoguenov, ${ }^{4}$ I. Sakelliou, ${ }^{2}$ \\ C. L. Carilli, ${ }^{5}$ C. S. Botzler,${ }^{6,7,8}$ M. Brusa,${ }^{4}$ N. Scoville, ${ }^{9,10}$ M. Ajiki, ${ }^{11}$ \\ P. Capak, ${ }^{9}$ L. Guzzo, ${ }^{12}$ G. Hasinger, ${ }^{4}$ C. Impey, ${ }^{13}$ K. JahnKe, ${ }^{2}$ \\ J. S. Kartaltepe, ${ }^{14}$ H. J. McCracken, ${ }^{15}$ B. Mobasher, ${ }^{16}$ T. Murayama, ${ }^{11}$ \\ S. S. SASAKI, ${ }^{11,17}$ Y. Shioya, ${ }^{17}$ Y. TANiguchi, ${ }^{17}$ and J. R. Trump ${ }^{13}$ \\ Received 2006 April 25; accepted 2006 August 15
}

\begin{abstract}
We have identified a complex galaxy cluster system in the COSMOS field via a wide-angle tail (WAT) radio galaxy, consistent with the idea that WAT galaxies can be used as tracers of clusters. The WAT galaxy, CWAT-01, is coincident with an elliptical galaxy resolved in the HST ACS image. Using the COSMOS multiwavelength data set, we derive the radio properties of CWAT-01 and use the optical and X-ray data to investigate its host environment. The cluster hosting CWAT-01 is part of a larger assembly consisting of a minimum of four X-ray luminous clusters within $\sim 2 \mathrm{Mpc}$ distance. We apply hydrodynamic models that combine ram pressure and buoyancy forces on CWAT-01. These models explain the shape of the radio jets only if the galaxy's velocity relative to the intracluster medium (ICM) is in the range of about 300$550 \mathrm{~km} \mathrm{~s}^{-1}$, which is higher than expected for brightest cluster galaxies (BCGs) in relaxed systems. This indicates that the CWAT-01 host cluster is not relaxed but is possibly dynamically young. We argue that such a velocity could have been induced through subcluster merger within the CWAT-01 parent cluster and/or cluster-cluster interactions. Our results strongly indicate that we are witnessing the formation of a large cluster from an assembly of multiple clusters, consistent with the hierarchical scenario of structure formation. We estimate the total mass of the final cluster to be approximately $20 \%$ of the mass of the Coma Cluster.
\end{abstract}

Subject headings: cosmology: observations - galaxies: individual (CWAT-01) — galaxies: jets radio continuum: galaxies — surveys - X-rays: galaxies: clusters

\section{INTRODUCTION}

Wide-angle tail (WAT) galaxies form a class of radio galaxies, usually found in clusters, whose radio jets have been bent into a wide $\mathrm{C}$ shape. The general morphology of WATs suggests that the sources interact significantly with their external environment. The most natural interpretation of the jet bending is that the jets are being swept back by ram pressure resulting from the high-velocity

\footnotetext{
1 Based on observations with the NASA/ESA Hubble Space Telescope, obtained at the Space Telescope Science Institute, which is operated by the Association of Universities for Research in Astronomy, Inc., under NASA contract NAS 5-26555; also based on observations obtained with $X M M-N e w t o n$, an ESA science mission with instruments and contributions directly funded by ESA member states and NASA; also based on data collected at the Subaru Telescope, which is operated by the National Astronomical Observatory of Japan; the European Southern Observatory, Chile; Kitt Peak National Observatory, Cerro Tololo Inter-American Observatory, and the National Optical Astronomy Observatory, which are operated by the Association of Universities for Research in Astronomy, Inc. (AURA), under cooperative agreement with the National Science Foundation; the National Radio Astronomy Observatory, which is a facility of the National Science Foundation operated under cooperative agreement by Associated Universities, Inc. Based also on observations obtained with MegaPrime/MegaCam, a joint project of the CanadaFrance-Hawaii Telescope (CFHT) and CEA/DAPNIA, at the CFHT, which is operated by the National Research Council (NRC) of Canada, the Institut National des Science de l'Univers of the Centre National de la Recherche Scientifique (CNRS) of France, and the University of Hawaii. This work is based in part on data products produced at TERAPIX and the Canadian Astronomy Data Centre.

2 Max-Planck-Institut für Astronomie, Königstuhl 17, Heidelberg, D-69117, Germany.

3 Fellow of the IMPRS for Astronomy and Cosmic Physics.

4 Max-Planck-Institut für Extraterrestrische Physik, D-85478 Garching, Germany.

5 National Radio Astronomy Observatory, P.O. Box 0, Socorro, NM 87801-0387.

${ }^{6}$ University of Auckland, Private Bag 92019, Morrin Road, Glen Innes, Auckland, New Zealand.
}

motion of the associated active elliptical galaxy through its surrounding intracluster medium (ICM), first developed by Begelman et al. (1979) and applied by a number of investigators (e.g., Burns 1981; Pinkney et al. 1994). In addition to ram pressure, buoyancy forces were introduced to explain the bending of the jets (e.g., Gull \& Northover 1973; Sakelliou et al. 1996). If the jet density is lower than the density of the surrounding medium, buoyancy forces will drag the jets toward regions of the ICM where the densities are equal.

A point first noticed by Burns (1981) was that WATs are usually associated with brightest cluster galaxies (BCGs; D or cD galaxies), which are expected to reside at rest at the bottom of the clusters' gravitational potential well (Quintana \& Lawrie 1982;

\footnotetext{
${ }^{7}$ University of Canterbury, Private Bag 4800, Christchurch, New Zealand.

${ }^{8}$ Universitäts-Sternwarte München, Scheinerstrasse 1, D-81679, München, Germany.

${ }^{9}$ California Institute of Technology, MC 105-24, 1200 East California Boulevard, Pasadena, CA 91125.

${ }^{10}$ Visiting Astronomer, University of Hawaii, 2680 Woodlawn Drive, Honolulu, HI, 96822.

${ }^{11}$ Astronomical Institute, Graduate School of Science, Tohoku University, Aramaki, Aoba, Sendai 980-8578, Japan.

${ }^{12}$ Osservatorio Astronomico di Brera, via Brera, Milan, Italy.

${ }^{13}$ Steward Observatory, University of Arizona, 933 North Cherry Avenue, Tucson, AZ 85721

${ }^{14}$ Institute for Astronomy, 2680 Woodlawn Drive, University of Hawaii, Honolulu, Hawaii, 96822.

${ }^{15}$ Institut d'Astrophysique de Paris, UMR 7095, 98 bis Boulevard Arago, 75014 Paris, France.

${ }^{16}$ Space Telescope Science Institute, 3700 San Martin Drive, Baltimore, MD 21218.

${ }_{17}$ Physics Department, Graduate School of Science and Engineering, Ehime University, 2-5 Bunkyo-cho, Matsuyama, Ehime 790-8577, Japan.
} 
Merritt 1984; Bird 1994). Thus, the large velocities of the WAT host galaxies relative to the ICM needed for the ram pressure models to shape the jets seemed to be inconsistent with velocities typical for BCGs. ${ }^{18}$ Therefore, it was necessary to invoke alternative scenarios to explain the bent shape of WAT galaxies (e.g., Eilek 1979). However, the most prominent explanation is that the jets are bent by ram pressure. It has been suggested in numerous studies that the necessary ram pressure may be provided during cluster mergers (e.g., Pinkney et al. 1994; Loken et al. 1995; Gomez et al. 1997; Sakelliou et al. 1996; Sakelliou \& Merrifield 2000). This merger scenario is consistent with cosmological models, such as the cold dark matter model (CDM), which propose that the structure in the universe is formed hierarchically with large features forming from mergers of smaller groups. The cluster potential well deepens then through accretion of poor clusters, dark matter, and gas into more massive systems. The material is accreted from supercluster filaments, which connect clusters into the large-scale structure of the universe (Evrard 1990; Jing et al. 1995; Frenk et al. 1996; Burns et al. 2002).

Based on ROSAT PSPC X-ray observations of a sample of nine Abell clusters containing WAT galaxies, Gomez et al. (1997) found evidence for statistically significant X-ray substructure in $90 \%$ (eight out of nine) of the clusters hosting WATs, as well as a strong correlation of the orientation of the jets and the direction of X-ray elongation within the core of the cluster. Combined with numerical hydro/ $N$-body simulations, their results are consistent with WAT clusters undergoing mergers with groups or subclusters of galaxies. Sakelliou \& Merrifield (2000) showed that WATs are not generally located at the centers of their host clusters as defined by their X-ray emission. They also found that the orientation of the bent jets is found to be preferentially pointed directly toward or away from the cluster center. Thus, if the morphology is due to ram pressure, WATs are then primarily on radial orbits through the cluster. These results are explained as a natural consequence of cluster mergers creating WAT galaxies (for details, see Sakelliou \& Merrifield 2000 and references therein). Blanton et al. (2001) presented optical imaging and spectroscopic observations of environments surrounding 10 bent radio sources. They found that the clusters display a range of line-of-sight velocity dispersions, $\sigma_{\|}$, from about 300 to $1100 \mathrm{~km} \mathrm{~s}^{-1}$. The upper limit of $\sigma_{\|}$suggests that the host clusters are either massive clusters and/or merging systems with significant substructure.

Since WATs are usually found in cluster environments, they can be used as efficient tools for cluster search, especially for high-redshift clusters, where we are biased by the dimming of galaxies in the optical and the ICM in X-ray emission. This approach has been successfully tested by Blanton et al. (2000, 2001, 2003) using the VLA ( Very Large Array) FIRST (Faint Images of the Radio Sky at Twenty centimeters; Becker et al. 1995) survey to search for galaxy clusters via WAT galaxies. The highest redshift cluster they have identified to date is at $z=0.96$ (Blanton et al. 2003).

In this paper we discuss the properties of a WAT radio galaxy (hereafter CWAT-01) found in the VLA-COSMOS $2 \mathrm{deg}^{2}$ survey, previously detected but not resolved by the NVSS (NRAO VLA Sky Survey; Condon et al. 1998) survey and not detected in the VLA FIRST survey (Becker et al. 1995). The multiwavelength

\footnotetext{
18 Malumuth et al. (1992) have shown that the velocity dispersion of the cD population is 0.3 of the dispersion of the cluster population; Beers et al. (1995) have found a velocity difference between the peculiar velocity of the central galaxy and the mean of the rest of the cluster galaxies $\leqslant 150 \mathrm{~km} \mathrm{~s}^{-1}$; recently, Oegerle et al. (2001), analyzing 25 Abell clusters, showed that peculiar velocities of cD galaxies differ only by $\sim 160 \mathrm{~km} \mathrm{~s}^{-1}$ from the mean cluster velocities.
}

data set of the COSMOS survey (Scoville et al. 2007a) enables us to use the radio data to derive the properties of this radio galaxy and the optical/X-ray data to investigate its host environment. In $\S 2$ we present the data used here. Section 3 describes the radio and optical properties of CWAT- 01 . In $\S 4$ we introduce the cluster and independently analyze its properties in the X-ray and the optical. We discuss the results in $\S 5$ and summarize them in $\S 6$.

For calculations in this paper, we assume $H_{0}=70, \Omega_{M}=0.3$, and $\Omega_{\Lambda}=0.7$. We define the synchrotron spectrum as $F_{\nu} \propto \nu^{-\alpha}$, with a positive spectral index, $\alpha>0$, throughout the paper.

\section{OBSERVATIONS AND DATA REDUCTION}

\subsection{Radio Data}

The $2 \mathrm{deg}^{2}$ COSMOS field was observed at $1.4 \mathrm{GHz}$ with the NRAO VLA in A and C configuration for a total time of $275 \mathrm{hr}$ (VLA-COSMOS survey; Schinnerer et al. 2007). The final $2 \mathrm{deg}^{2}$ map has a typical rms of $\sim 10.5(15) \mu \mathrm{Jy}_{\text {beam }}{ }^{-1}$ in the inner $1(2) \mathrm{deg}^{2}$, with a resolution of $1.5^{\prime \prime} \times 1.4^{\prime \prime}$, thus making it the largest contiguous area covered in the radio-wavelengths regime with such a high sensitivity. The VLA-COSMOS Large Project catalog (presented in Schinnerer et al. 2007) contains $\sim 3600$ radio sources, $\sim 90$ of which are clearly extended (most of them double-lobed radio galaxies). The sensitivity of the survey combined with the high resolution and the large area coverage makes the VLA-COSMOS project extremely valuable for, e.g., studies of the submillijansky radio population, dust-obscured star formation history of the universe, and evolution of radio-loud active galactic nuclei (AGNs). The survey used the standard VLA L-band continuum frequencies and the multichannel continuum mode. The complete data reduction was performed using the standard VLA software AIPS. The A- and C-array data were combined in the $u v$ plane and then imaged using the task IMAGR. Cleaning boxes around bright sources were defined manually; the two intermediate frequencies (IFs) and the left and right polarization were imaged separately and then combined into the final map. For more information about the survey and its scientific objectives see Schinnerer et al. (2007). The local rms noise in the mosaic around CWAT-01 is $\sim 10.5 \mu \mathrm{Jy}_{\text {beam }}{ }^{-1}$.

Subsequent observations of CWAT-01 were obtained in 2005 June with the VLA in the CnB configuration at $4.8 \mathrm{GHz}$. The standard C-band continuum frequencies were used, and the observations were performed in the standard continuum mode. The CWAT-01 field was observed for 40 minutes on source. After flux and phase calibration the data set was imaged using the AIPS task IMAGR. The IFs and the left and right polarization were imaged together. Clean boxes were defined manually, the number of CLEAN iterations in IMAGR was set to 3000, and the flux cutoff to $60 \mu \mathrm{Jy}$. The $u v$ data points were weighted using the natural weighting function (UVWTFN was set to "N"). Due to the asymmetric $u v$ coverage the resolution of the $4.8 \mathrm{GHz}$ map was tapered down to obtain a rounder beam (the UVTAPER option in IMAGR was set to $35 \mathrm{k} \lambda$ ). The resolution and rms noise in the tapered map are $7.27^{\prime \prime} \times 5.53^{\prime \prime}$ and $\sim 40 \mu \mathrm{Jy}_{\text {beam }}{ }^{-1}$, respectively.

\subsection{X-Ray Data}

The COSMOS $2 \mathrm{deg}^{2}$ field is being observed by the XMMNewton satellite (Jansen et al. 2001) for an awarded time of $1.4 \mathrm{Ms}$ (Hasinger et al. 2007). The data collected to date amount to $0.8 \mathrm{Ms}$ over the $2 \mathrm{deg}^{2}$ area with an effective depth of $\sim 40 \mathrm{ks}$, taking vignetting into account. In this study we use some of the results of Finoguenov et al. (2007), who identified clusters in the COSMOS field via diffuse X-ray emission. The flux limit for the cluster identification in the $0.5-2 \mathrm{keV}$ energy band is $2 \times 10^{-15} \mathrm{ergs} \mathrm{cm}^{-2} \mathrm{~s}^{-1}$. 
TABLE 1

Specifications of Galaxies within the Cluster Area that Have IMACS Spectra

\begin{tabular}{ccccc}
\hline \hline Name & R.A. (J2000.0) & Decl. (J2000.0) & Type & $z_{\text {spec }} \pm \Delta z_{\text {spec }}$ \\
\hline COSMOS J100021.81+022328.5 …...... & 100021.816 & +022328.523 & Elliptical & $0.22067 \pm 0.00007$ \\
COSMOS J100025.30+022522.5 ........... & 100025.298 & +022522.476 & Elliptical & $0.22090 \pm 0.00013$ \\
\hline
\end{tabular}

Notes.-Units of right ascension are hours, minutes, and seconds, and units of declination are degrees, arcminutes, and arcseconds. For details about IMACS, see Trump et al. 2007). The last column specifies the spectroscopic redshift and error.

${ }^{a}$ Corresponds to SDSS J100021.81+022328.46 (see Table 2).

Finoguenov et al. (2007) reported four diffuse structures within $4^{\prime}$ of CWAT-01, one of them containing CWAT-01. We perform the spectral analysis of the extended emission associated with the four identified clusters in the following way. The EPIC pn observations of the COSMOS field have been merged together, and a uniform cleaning criterion for background flares has been subsequently applied. Observations that do not satisfy this criterion are removed. In this way we achieve a uniform background level for the clean data set. The resulting file consists of a total of 365 ks homogeneously cleaned data (for the whole $2 \mathrm{deg}^{2}$ field). However, the region around CWAT- 01 is still covered at an effective depth of $43 \mathrm{ks}$, as the removed observations are all located at the edge of the COSMOS field.

Since the instrumental background is not uniform over the detector (Lumb et al. 2002), in order to estimate the background we produce a background file from the same merged event file by excluding the area containing a detected X-ray source. This removes $\sim 20 \%$ of the area, which we account for in correcting for the background. The background is further assumed to be the same in detector coordinates. In calculating the background spectrum, the sky position of the cluster is mapped to the detector, taking into account multiple pointings, which map the same region of the sky on different detector areas. The background spectrum is collected weighting accordingly the contribution in each detector pixel. For the clusters in this study the ratio of the background to signal accumulation times is 5 , which is sufficient to reduce the statistical uncertainty associated with the background subtraction. The calculation of auxiliary response files is performed by the SAS-based task clarf of Finoguenov et al. (2004), which takes the mosaicking into account. The pn calibrations involved in the data reduction correspond to SAS (Science Analysis Software; Watson et al. 2001), version 6.5.

\subsection{Optical Data}

The optical imaging data of the $2 \mathrm{deg}^{2}$ COSMOS field that we use in this paper was obtained in the spring of 2004 and 2005. Within the COSMOS HST Treasury project the $2 \mathrm{deg}^{2}$ field was imaged in 590 orbits during Cycles 12 and 13 using the Advanced Camera for Surveys (ACS; Scoville et al. 2007b). The F814W band imaging has a $0.07^{\prime \prime}$ resolution and a $10 \sigma$ sensitivity of $I_{\mathrm{AB}}=27.2$. Each of the 590 fields consists of four exposures, which are calibrated and combined into a single image for each field using the MultiDrizzle software (for details, see Koekemoer et al. 2007). The whole COSMOS field was imaged with the Suprime-Cam camera (at the Subaru telescope) in six broadband filters, $B_{J}, V_{J}, g^{+}, r^{+}, i^{+}$, and $z^{+}$, with $5 \sigma$ sensitivity in AB magnitudes of 27.3, 26.6, 27.0,26.8, 26.2, and 25.2, respectively (Taniguchi et al. 2007; Capak et al. 2007). The $u^{*}$ - and $i^{*}$-band images of the whole $2 \mathrm{deg}^{2}$ COSMOS field were obtained with the CFHT (Canada-France-Hawaii Telescope). The $5 \sigma$ sensitivity in $\mathrm{AB}$ magnitudes is 26.4 and 24.0 for $u^{*}$ and $i^{*}$, respectively.

The COSMOS photometric catalog was constructed using the Subaru $i^{+}$-band image. The details on constructing the photometric redshift catalog are described in Mobasher et al. (2007). The catalog produces photometric redshifts accurate to $d z /(1+z)=$ 0.034 . We use these redshifts to study the optical (sub)structure of the clusters in later sections.

\subsection{Redshift}

Trump et al. (2007) presented spectroscopic redshifts of the first $\sim 500$ X-ray- and radio-selected targets in the $2 \mathrm{deg}^{2}$ COSMOS field. The spectra were obtained using the Magellan IMACS instrument. They also perform a robust classification of the observed objects (for details, see Trump et al. 2007 and references therein). In the cluster area around CWAT-01 (see $\S$ 4.2.2) there are two galaxies that have IMACS spectra. Their properties, classification, and redshifts are reported in Table 1. Searching for high-resolution $(R=1800)$ Sloan Digital Sky Survey (SDSS; York et al. 2000; Abazajian et al. 2003, 2004, 2005; Adelman-McCarthy et al. 2006) spectra, we find that three galaxies in our cluster area around CWAT-01 have SDSS spectra. One of them was observed with IMACS. The specifications of these galaxies are listed in Table 2. It is worth noting that the galaxies with spectroscopic redshifts are the most prominent galaxies within each cluster (see $\S 4$ ).

The mean spectroscopic redshift of the galaxies presented above is 0.2209 with an accuracy of $2.8 \times 10^{-4}$. Given the dispersion of the photometric redshift values they are compatible with a redshift of $z=0.22$. Therefore, for the scope of this paper we adopt a mean cluster redshift of $z=0.22$, based on four measured spectroscopic redshifts.

\section{THE WIDE-ANGLE TAIL GALAXY: CWAT-01}

The radio galaxy first resolved in the VLA-COSMOS survey, CWAT-01, has a morphology typical for WAT galaxies. Its radio jets are bent into a wide $\mathrm{C}$ shape (see Fig. 1 for an example). In $\S 3.1$ we describe the structure of CWAT- 01 and derive its radio properties in order to investigate the correlation to its host environment in latter sections. In $\S 3.2$ we describe the optical properties of the CWAT-01 host galaxy.

\subsection{Radio Properties of CWAT-01}

\subsubsection{The Structure of CWAT-01}

The radio galaxy discussed here was first detected in the NVSS survey (NVSS J100027+022104; Condon et al. 1998), but not resolved due to the low resolution of the NVSS $\left(45^{\prime \prime}\right)$. It was first resolved in the $1.4 \mathrm{GHz}$ mosaic of the central $1 \mathrm{deg}^{2}$ field from the VLA-COSMOS Pilot Project (Schinnerer et al. 2004). CWAT-01 was not detected in the FIRST survey as this survey overresolves radio sources larger than $\sim 10^{\prime \prime}$ (and thus underestimates their fluxes; see Becker et al. 1995 for details). Hence, a galaxy like CWAT-01, which extends over more than $1^{\prime}$ on the plane of the sky and has a total flux density of $\sim 13 \mathrm{mJy}$ (see below), would be strongly resolved out in the FIRST survey; i.e., the galaxy would consist of multiple components and the flux from the extended regions ( $\left(10^{\prime \prime}\right)$ of each component would be missed. The 
TABLE 2

Specifications of Galaxies within the Cluster Area that Have SDSS Spectra

\begin{tabular}{cccccc}
\hline \hline Name & Plate & Fiber & R.A. (J2000.0) & Decl. (J2000.0) & $z_{\text {spec }} \pm \Delta z_{\text {spec }}$ \\
\hline SDSS J10004.35+022550.71................. & 501 & 348 & 10004.354 & +022550.711 & $0.2201 \pm 0.0001$ \\
SDSS J10006.65+022225.98............... & 501 & 353 & 10006.654 & +022225.982 & $0.2221 \pm 0.0002$ \\
SDSS J100021.81+022328.46 …........... & 501 & 388 & 100021.815 & +022328.463 & $0.2206 \pm 0.0001$ \\
\hline
\end{tabular}

Notes.-Units of right ascension are hours, minutes, and seconds, and units of declination are degrees, arcminutes, and arcseconds. The last column specifies the spectroscopic redshift and error.

${ }^{\text {a }}$ Corresponds to COSMOS J100021.81+022328.5 (see Table 1).

individual parts of CWAT-01 that could have been detected by FIRST have flux densities below the detection limit of the FIRST survey $(1 \mathrm{mJy})$. Thus, CWAT-01 or any fraction of the galaxy stays undetected in the FIRST survey.

Our new VLA-COSMOS observations, as part of the VLACOSMOS Large Project (Schinnerer et al. 2007), at 1.4 GHz provided significantly better data of this extended radio galaxy. The new 1.4 GHz VLA-COSMOS map of CWAT-01 is shown in Figure 1 (the resolution is $1.5^{\prime \prime} \times 1.4^{\prime \prime}$ ). The radio jets of the galaxy are curved in a $\mathrm{C}$ shape, typical for WAT galaxies. The central radio peak of CWAT-01 is at $\alpha=10^{\mathrm{h}} 00^{\mathrm{m}} 27.35^{\mathrm{s}}$ and $\delta=+02^{\circ} 21^{\prime} 24.15^{\prime \prime}$ (J2000.0), and its host galaxy is an elliptical galaxy with a photometric redshift of $z=0.2 \pm 0.03$. The photometric redshift was taken from the COSMOS photometric redshift catalog described in detail in Mobasher et al. (2007). The quoted error is the $1 \sigma$ error obtained from the $95 \%$ confidence interval. The optical counterpart is discussed in more detail in $\S 3.2$. The jets are barely resolved in width (i.e., they are perpendicular to the jet axis) out to points E1 and W1 (see Fig. 1). The eastern radio jet can be traced out to a projected distance of $\sim 1^{\prime}(\sim 210 \mathrm{kpc})$ from the central galaxy. At point $\mathrm{E} 1$ it bends to the west (in the projected plane) and broadens. The end of the jet is marked with E2 (see Fig. 1). The structure of the western jet is more complex: It extends to a distance of $\sim 45^{\prime \prime}(\sim 160 \mathrm{kpc})$ in the plane of the sky. From the core until W1 it is narrow, but it indicates curvature in the northern part. After W1 the jet broadens and bends slightly to the west in the projected plane (W2). The faint feature labeled as W3 in Figure 1 seems also to be part of CWAT-01. The integrated flux density of CWAT-01 at $1.4 \mathrm{GHz}$ is $12.69 \mathrm{mJy}$. This is within the errors of the reported NVSS flux density of $13.5 \pm 1.9 \mathrm{mJy}$.

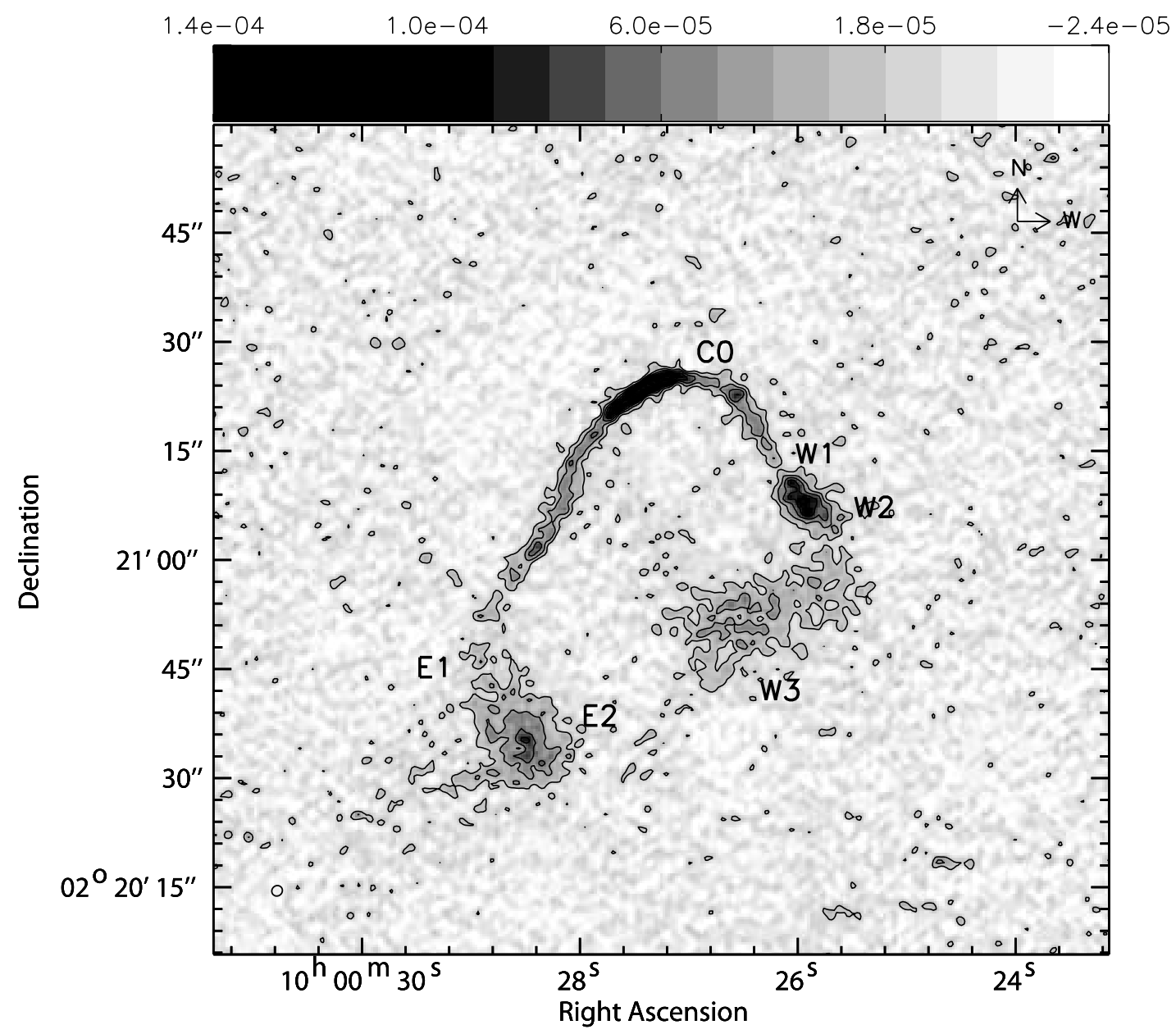

FIG. 1.-A $1.4 \mathrm{GHz}$ radio map of the wide-angle tail galaxy CWAT-01 in the COSMOS field in gray scale with contours overlaid. The contour levels are in steps of $2 \sigma$

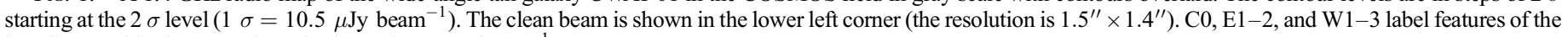
jets discussed in the text. The color bar units are Jy beam ${ }^{-1}$. 


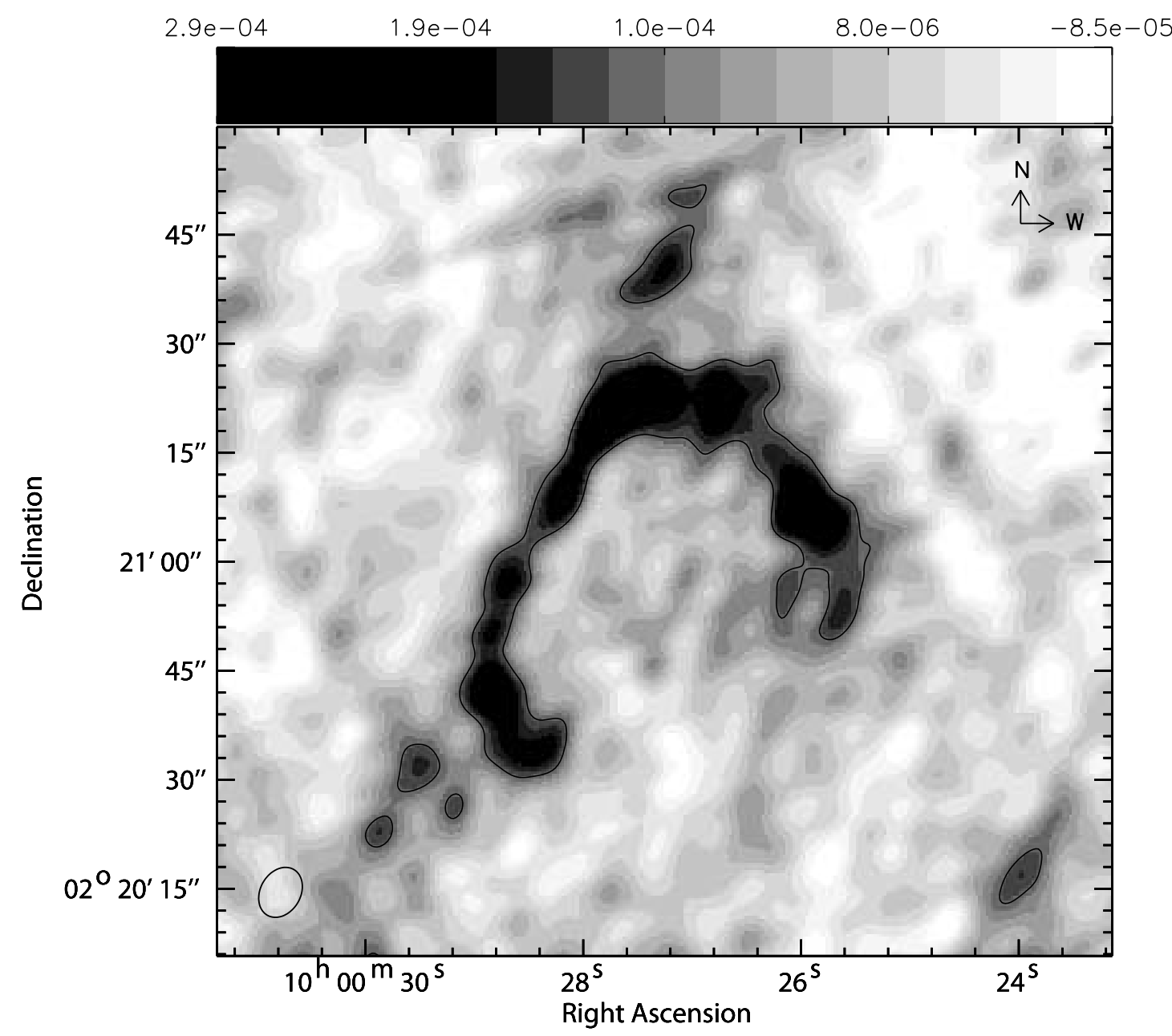

FIG. 2.-A $4.8 \mathrm{GHz}$ radio map of CWAT-01 with contours overlaid. The contour levels are in steps of $2 \sigma$ starting at the $3 \sigma$ level $\left(1 \sigma=40 \mu \mathrm{Jy}\right.$ beam $\left.{ }^{-1}\right)$. The clean beam is shown in the lower left corner (the resolution is $7.27^{\prime \prime} \times 5.53^{\prime \prime}$ ). The color bar units are $\mathrm{Jy} \mathrm{beam}^{-1}$.

For consistency we use the flux density derived from the VLACOSMOS for calculations throughout the paper.

The bending angle of the jets, which we define as the angle between lines parallel to the part of the jet closest to the core (see Fig. 11, bottom), is $\sim 100^{\circ}$. The asymmetry of the jets may be due to projection effects, which would indicate that the whole structure is not moving only in the plane of the sky. However, with the data in hand we cannot reach any firm conclusions about projection effects nor rule them out. More radio bands and higher resolution radio data where relativistic core beaming effects could be explored might resolve this issue; e.g., on $\sim 10 \mathrm{kpc}$ scales from the radio core of the galaxy (where the bulk motion of the particles is relativistic) relativistic effects yield that the ratio of the radio brightness of the two jets can be correlated with the orientation angle.

\subsubsection{Spectral Index}

The $4.8 \mathrm{GHz}$ map at $7.27^{\prime \prime} \times 5.53^{\prime \prime}$ resolution is shown in Figure 2. The main features seen in the $1.4 \mathrm{GHz}$ map (with a resolution of $1.5^{\prime \prime} \times 1.4^{\prime \prime}$; Fig. 1) are still apparent, although the much lower resolution reduces the amount of details. The total integrated flux at $4.8 \mathrm{GHz}$ is $4.1 \mathrm{mJy}$. To obtain the spectral index map (shown in Fig. 3), the $1.4 \mathrm{GHz}$ image was convolved to the resolution of the $4.8 \mathrm{GHz}$ map, and the two images were regridded to the same pixel scale. Pixels with values below $3 \sigma$ in each map were blanked. The middle of the central feature is a flat-spectrum region $(0.1 \lesssim \alpha \lesssim 0.3)$, with the spectral index steepening to $\alpha \sim 1$ to the northwest edge in this feature. The outer region (corresponding to $\mathrm{E} 1)$ with a flat spectrum $(0.2 \lesssim \alpha \lesssim 0.6)$ suggests possible reacceleration regions. The spectral index steepens to $\alpha \sim 1$ at $\mathrm{E} 2$. In $\mathrm{W} 2$ the spectrum emission is on average steeper than in the E1 feature, with an average spectral index corresponding to $\alpha \sim 0.7$. The mean spectral index in the total source is $\alpha=0.6$, which we use for calculations throughout this paper unless mentioned otherwise.

\subsubsection{Radio Power and Luminosity}

We compute the radio power of CWAT- 01 at $1.4 \mathrm{GHz}$ using a spectral index of $\alpha=0.6$ (see $\S 3.1 .2$ ) and the luminosity distance $(1.1 \mathrm{Gpc})$ at $z=0.22$. The radio power of CWAT- 01 is then $P_{1.4}=2.0 \times 10^{24} \mathrm{~W} \mathrm{~Hz}^{-1}$, which places the radio galaxy between FR I and FR II type galaxies where WATs are normally found (Hardcastle \& Sakelliou 2004).

We also calculate the total radio luminosity, given by (e.g., O’Dea \& Owen 1987)

$$
\begin{aligned}
L_{\mathrm{tot}}= & 1.2 \times 10^{27} D_{L,[\mathrm{Mpc}]}^{2} F_{0} \nu_{0}^{\alpha}(1+z)^{-(1-\alpha)} \\
& \times(1-\alpha)^{-1}\left(\nu_{2}^{1-\alpha}-\nu_{1}^{1-\alpha}\right)\left[\mathrm{ergs} \mathrm{s}^{-1}\right],
\end{aligned}
$$

where $D_{L,[\mathrm{Mpc}]}$ is the luminosity distance expressed in Mpc, and $F_{0}$ is the flux density, at a fiducial frequency $\nu_{0}$, expressed in Jy. We take the lower and upper frequencies to be $\nu_{1}=10 \mathrm{MHz}$ and 


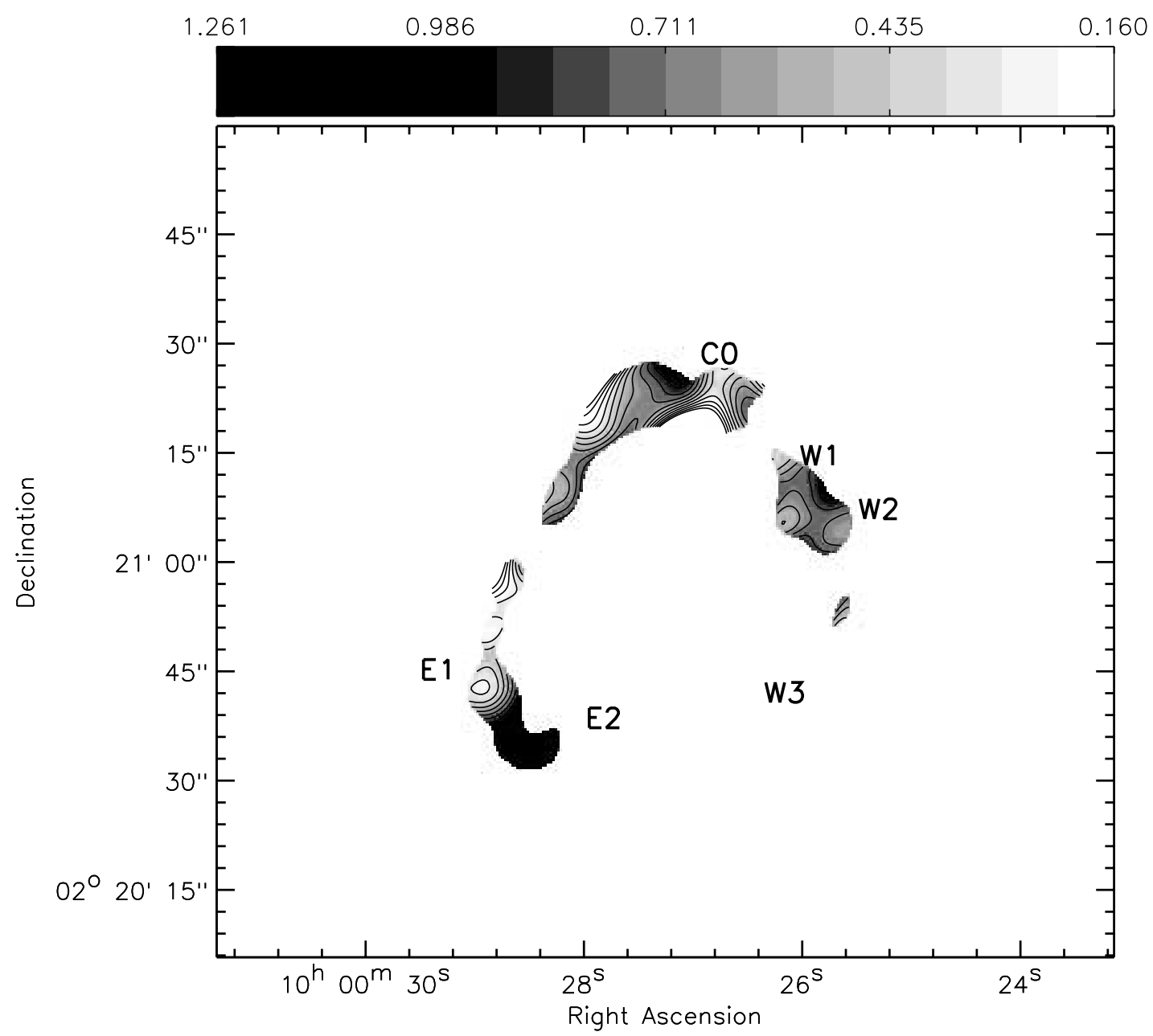

FIG. 3.- Spectral index map of CWAT-01 for pixels with values $>3 \sigma$ in both maps (1.4 and $4.8 \mathrm{GHz})$. C0, E1-2, and W1-3 are labeled as in Fig. 1 and are presented here for clarity. We define the spectral index as $F_{\nu} \propto \nu^{-\alpha}$ throughout the paper. The contour levels are $0.1,0.2,0.3$, etc.

$\nu_{2}=100 \mathrm{GHz}$, respectively. The observed frequency is $\nu_{0}=$ $1.4 \mathrm{GHz}$. The total luminosity of CWAT- 01 is then $L_{\mathrm{tot}}=3.2 \times$ $10^{41} \mathrm{ergs} \mathrm{s}^{-1}$, typical for peculiar radio galaxies (Pacholczyk 1970).

\subsubsection{Magnetic Field and Minimum Pressure}

Assuming that the total energy in a radio galaxy is the sum of the energy of electrons, $E_{e}$, heavy particles, $E_{p}$, and the magnetic field, $E_{B}, E_{\text {tot }}=E_{e}+E_{p}+E_{B}$, we can estimate the minimum energy density, $u_{\text {me }}$, and the corresponding magnetic field, $B_{\text {me }}$, using the minimum energy condition, which corresponds almost to equipartition between the relativistic particles and the magnetic field. ${ }^{19}$ We adopt the expression from Miley (1980; for details, see Pacholczyk 1970):

$$
\begin{aligned}
u_{\mathrm{me}}= & \frac{7}{3} \frac{B_{\mathrm{me}}^{2}}{8 \pi}\left[\mathrm{dyn} \mathrm{cm}^{-2}\right], \\
B_{\mathrm{me}}= & 5.69 \times 10^{-5}\left[\frac{1+k}{\eta}(1+z)^{3+\alpha}\right. \\
& \left.\times \frac{1}{\Theta_{x} \Theta_{y} l \sin ^{3 / 2} \Phi} \frac{F_{0}}{\nu_{0}^{\alpha}} \frac{\nu_{2}^{1 / 2-\alpha}-\nu_{1}^{1 / 2-\alpha}}{(1 / 2)-\alpha}\right]^{2 / 7} \quad[\mathrm{G}],
\end{aligned}
$$

19 Equipartition requires that the magnetic energy is equal to the total particle energy, i.e., $E_{B}=E_{e}+E_{p}$, while the minimum energy condition holds for $E_{B}=$ $(3 / 4)\left(E_{e}+E_{p}\right)$. Hence, the computed total energy, $E_{\mathrm{tot}}=E_{e}+E_{p}+E_{B}$, agrees within $\sim 10 \%$ for the first and latter. where $k$ is the ratio of relativistic proton to relativistic electron energies, $\eta$ is the filling factor of the emitting region, $z$ is the redshift, $\Theta_{x}$ and $\Theta_{y}$ correspond to the clean beam widths, $l$ is the pathlength through the source along the line of sight, $\Phi$ is the angle between the uniform magnetic field and the line of sight, $F_{0}$ is the flux density at a fiducial frequency $\nu_{0}, \nu_{1}$ and $\nu_{2}$ are the lower and upper frequency cutoffs, and $\alpha$ is the spectral index. The minimum energy density and the corresponding magnetic field were measured in the middle of the diffuse portion of the eastern radio jet in the $1.4 \mathrm{GHz}$ map with the following assumptions: (1) the radio plasma fills the volume completely $(\eta=1)$, (2) the magnetic field is transverse to the line of sight $(\sin \Phi=1)$, (3) the relativistic proton energy equals the relativistic electron energy $(k=1)$, (4) there is cylindrical symmetry, and (5) the radio spectrum spans from $10 \mathrm{MHz}$ to $100 \mathrm{GHz}$. The mean spectral index derived from the spectral index map in this part of the jet corresponds to $\alpha=0.65$. The resulting magnetic field is $B_{\mathrm{me}}=3.7 \mu \mathrm{G}$, and the minimum energy density is $u_{\mathrm{me}}=1.3 \times$ $10^{-12}$ dyn $\mathrm{cm}^{-2}$. The minimum internal pressure within the jets is then $P_{\min }=u_{\mathrm{me}} / 3=4.3 \times 10^{-13} \mathrm{dyn} \mathrm{cm}^{-2}$.

\subsubsection{The Particle Lifetime}

The synchrotron age of the electrons at frequency $\nu$ is given by van der Laan \& Perola (1969) assuming the following model. The electrons age as a result of synchrotron and inverse Compton losses due to the interaction with the cosmic microwave background 
(CMB). There is a brief "generation phase," during which the relativistic gas is presumably created by the active galaxy, and a long-term "remnant phase" during which the particle supply is switched off. The model computes the lifetime of the "remnant phase" as it assumes that the lifetime of the "generation phase" is much shorter:

$$
t \sim 2.6 \times 10^{4} \frac{B^{1 / 2}}{\left(B^{2}+B_{R}^{2}\right)[(1+z) \nu]^{1 / 2}}[\mathrm{yr}]
$$

where $B$ is the magnetic field in the jet and $B_{R}$ is the equivalent magnetic field of the CMB radiation, $B_{R}=4(1+z)^{4} \mu \mathrm{G}$. In order to constrain the electron lifetime, we substitute into equation (4) the magnetic field corresponding to the minimum energy condition, $B=B_{\text {me }}$, calculated for the region at the end of the radio jets at $1.4 \mathrm{GHz}$ (E2 and W2 in Fig. 1). The magnetic field $B_{\mathrm{me}}$ is again derived, making the same assumptions as in $\S 3.1 .4$. The mean spectral index at the ends of the eastern and western jets corresponds to 0.9 and 0.7 , respectively, and the minimum-energy magnetic fields are then 3.5 and $3.1 \mu \mathrm{G}$, respectively. Both values give the lifetime of an electron radiating at $1.4 \mathrm{GHz}$ of $\sim 13 \mathrm{Myr}$. Therefore, if we assume that there is no particle reacceleration within the jets, the relativistic electrons created in or near the core could travel the whole jet length within their lifetime with bulk velocities in the range of about $(0.04-0.05) c$.

\subsection{The Host Galaxy}

CWAT-01 is coincident with an elliptical galaxy, shown in Figure 4, located at $\alpha=10^{\mathrm{h}} 00^{\mathrm{m}} 27.43^{\mathrm{s}}$ and $\delta=+02^{\circ} 21^{\prime} 23.62^{\prime \prime}$ (J2000.0). The spectral energy distribution (SED) type, reported in the COSMOS photometric redshift catalog (Mobasher et al. 2007; Capak et al. 2007) is 1.33 (ellipticals and $\mathrm{Sa} / \mathrm{Sb}$ correspond to 1 and 2, respectively; see Coleman et al. [1980] and Kinney et al. [1996] for details). The photometric redshift of the galaxy is $z=0.2 \pm 0.03$ (the quoted error is the $1 \sigma$ error obtained from the $95 \%$ confidence interval). We construct the surface brightness profile of the host galaxy (shown in Fig. 5) using the GIPSY ellipse-fitting task ELLFIT on the background-subtracted HST ACS F814W-band image (Koekemoer et al. 2007). The surface brightness follows the $r^{1 / 4}$ law fairly well, but it deviates from it in the outer parts, indicating an excess in surface brightness, possibly an extended halo (Fig. 5, dashed line). The early-type morphology, the extensive envelope, and the shallower surface brightness profile compared to the $r^{1 / 4}$ law suggest that the CWAT01 host galaxy might be classified as a D-type galaxy (e.g., Beers \& Geller 1983). To obtain a better fit to the surface brightness, we fit the Sersic model $I(r)=I_{\text {eff }} e^{b_{n}\left[1-\left(r / r_{\text {eff }}\right)^{1 / n}\right]}, b_{n} \sim 2 n-0.324$ (Sersic 1968), to the data with effective radius, $r_{\text {eff }}$, effective intensity, $I_{\text {eff }}$, and Sersic index, $n$, as free parameters (Fig. 5, solid line). The data are very well fit by the Sersic law with $n=5.0$, $r_{\text {eff }}=8.2 \mathrm{kpc}$, and an effective surface brightness of $\mu_{\text {eff }}=$ $-2.5 \log I_{\text {eff }}-48.6=22.1 \mathrm{mag} \operatorname{arcsec}^{-2}$. It has already been noted by Schombert (1987) that intrinsically bright ellipticals are flatter (i.e., have higher values of $n$ ) and that intrinsically faint ellipticals have more curvature (i.e., lower values of $n$ ) than predicted by the $r^{1 / 4}$ law. Typical values of $n$ for BCGs are $n>4$ (Graham et al. 1996). The effective radius and the Sersic index of the CWAT-01 host galaxy make it consistent with being a $\mathrm{BCG}$ at the low end of the $n$ versus $r_{\text {eff }}$ correlation for BCGs (for details, see Graham et al. 1996). In $\S 4.2 .2$ we show that the CWAT-01 host galaxy is indeed the brightest galaxy in its parent cluster.
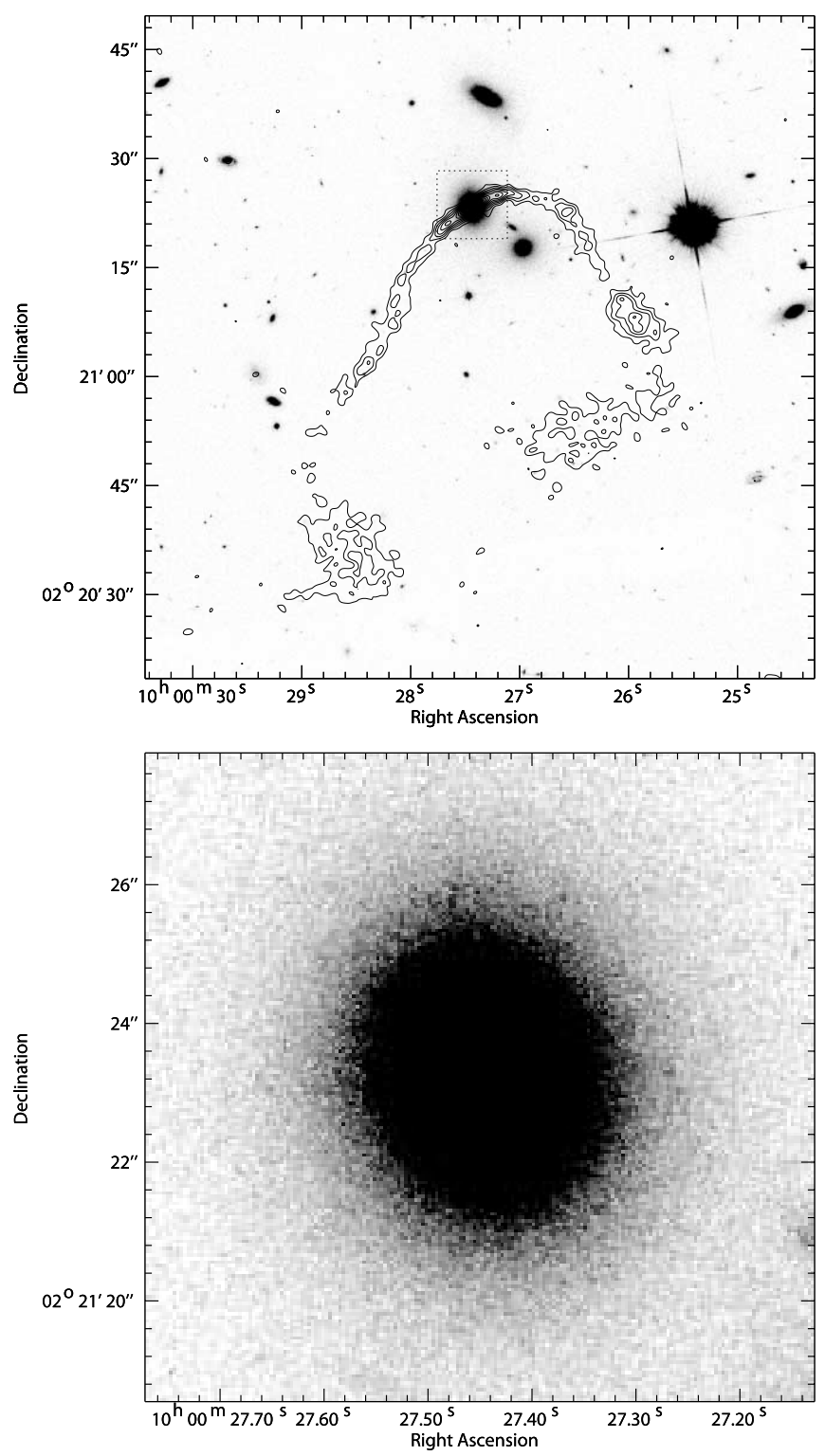

FIG. 4.-Top: HST ACS F814W-band (gray scale) image of the CWAT-01 host galaxy with $1.4 \mathrm{GHz}$ radio contours overlaid. The contour levels are in steps of $2 \sigma$ starting at the $3 \sigma$ level $\left(1 \sigma=10.5 \mu \mathrm{Jy}_{\text {beam }}{ }^{-1}\right)$. The dashed box indicates the region shown in the bottom panel. Bottom: HST ACS F814W-band image of the CWAT-01 host galaxy. The galaxy has the morphology of an elliptical galaxy.

\section{THE CLUSTERS}

WATs are normally found in cluster environments and are in general associated with the BCG. The X-ray image of the field around CWAT-01 (described in $\S 2.2$ ) showed the presence of multiple extended X-ray sources around the WAT. In addition, in $\S 3.2$ we have shown that the CWAT-01 host galaxy has the characteristics of BCGs. Taking advantage of the availability of the COSMOS multiwavelength data set we investigate the nature and properties of CWAT-01's environment in this section.

The whole cluster structure detected via diffuse X-ray emission is part of the large-scale structure component, LSS 17, reported by Scoville et al. (2007b). The diffuse X-ray emission shows substructure itself. Throughout the paper we use the following nomenclature. We refer to the whole area identified via diffuse X-ray emission as a "cluster assembly." The cluster assembly encompasses four subclumps (i.e., four individual diffuse X-ray emitting 


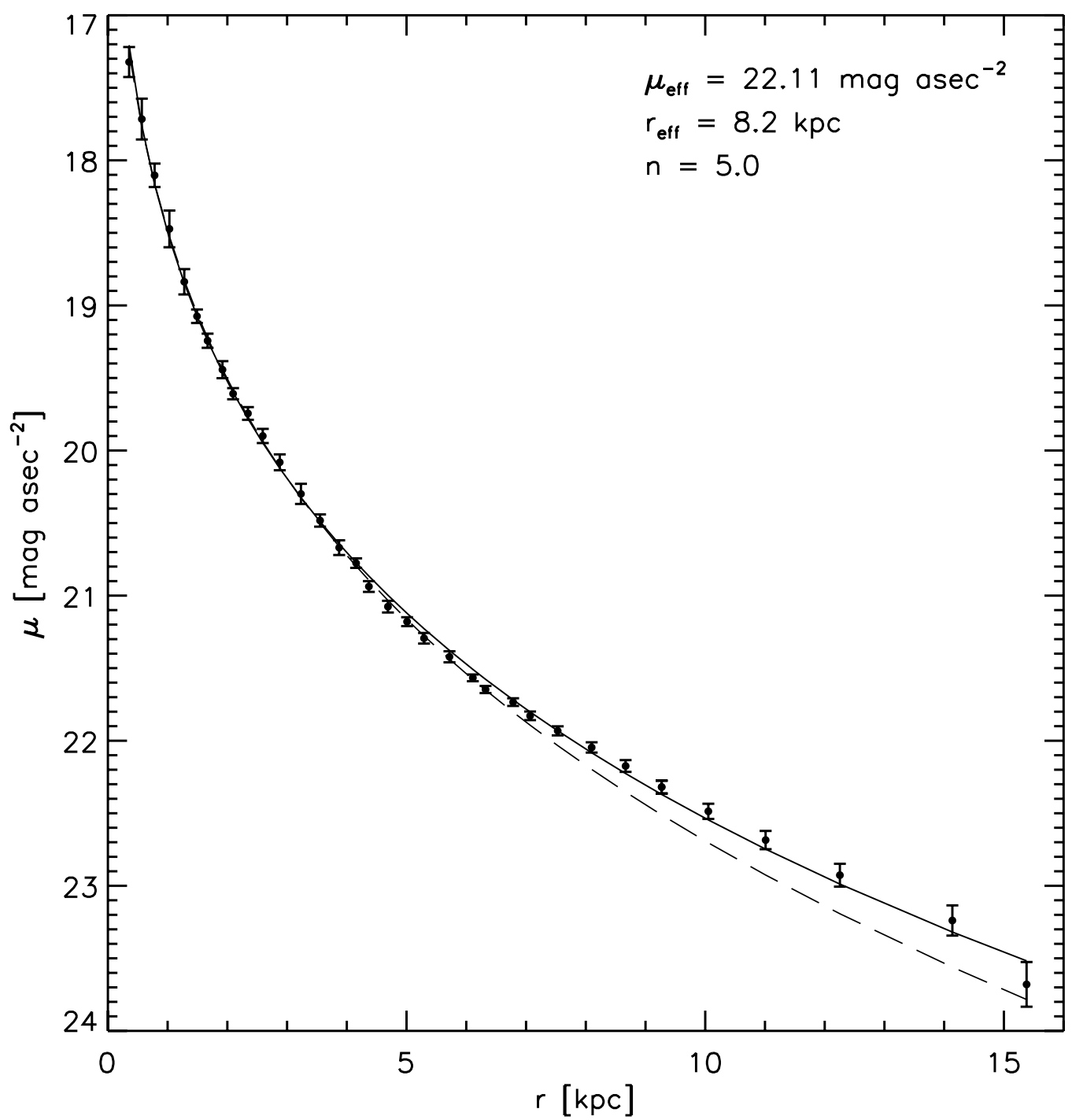

FIG. 5.-Surface brightness profile of the optical counterpart of CWAT-01. The surface brightness is expressed in AB magnitudes and was derived from the HST ACS F814W-band image (see text for details). The dots are the data points with $1 \sigma$ error bars. The de Vaucouleurs fit (dashed line) reveals an excess in surface brightness in the outer parts of the galaxy, while the Sersic law fits the profile very well (solid line). The effective surface brightness, effective radius, and Sersic index for the redshift of $z=0.22$ are listed at the top right.

regions), which we call "clusters" or "poor clusters," and is embedded in LSS 17; see, for example Figure 6 (top).

\subsection{X-Ray Properties}

The search for extended X-ray sources in the COSMOS $2 \mathrm{deg}^{2}$ field (for details, see Finoguenov et al. 2007) reveals four diffuse sources within $4^{\prime}$ radius of CWAT-01. Figure 6 (top) shows a part of the X-ray image in the $0.5-2 \mathrm{keV}$ band encompassing the cluster assembly. In the middle panel of Figure 6 we display the same area in the sky, but from the wavelet reconstruction of the $0.5-2 \mathrm{keV}$ band image that was presented in Finoguenov et al. (2007) and used for their cluster search. CWAT-01 is located in the southeastern cluster (hereafter the CWAT-01 parent cluster).

Finoguenov et al. (2007) assigned redshifts to the identified diffuse X-ray sources by analyzing redshift bins of width $\Delta z=$ 0.2 using the COSMOS photometric redshift catalog (Mobasher et al. 2007). Three of the four diffuse X-ray sources described here are associated with a large galaxy concentration in the same redshift bin with the median photometric redshifts of the clusters of 0.22 (Finoguenov et al. 2007). A description of the cluster
$\mathrm{X}$-ray catalog names, their positions, and their fluxes is given in Table 3. In the next sections we show that the fourth diffuse X-ray source can also be associated with an overdensity at a redshift of about $z=0.22$. For the purpose of this paper we assume that the clusters are all located at the same redshift and calculate their properties at $z=0.22$.

\subsubsection{The Cluster Assembly}

For the spectral analysis we used the energy band of 0.5$3 \mathrm{keV}$, since the counts at energies above $3 \mathrm{keV}$ are dominated by background photons. Nonetheless, energies above $3 \mathrm{keV}$ can be used to check the quality of the background subtraction, and this was found to be satisfactory. Based on the surface brightness level, five main zones were constructed for the spectral analysis in such a way to avoid bright point sources. The zones are shown in Figure 6 (bottom). Zones 1 (which corresponds to the CWAT-01 parent cluster), 3 , and 4 are sampled with three subzones, labeled $\mathrm{a}, \mathrm{b}$, and $\mathrm{c}$. Table 4 summarizes the results of the spectral analysis based on the APEC thermal-emission model (Smith et al. 2001). We report the temperature, normalization, and their corresponding errors, the reduced $\chi^{2}$ value, and the number of degrees of freedom, 


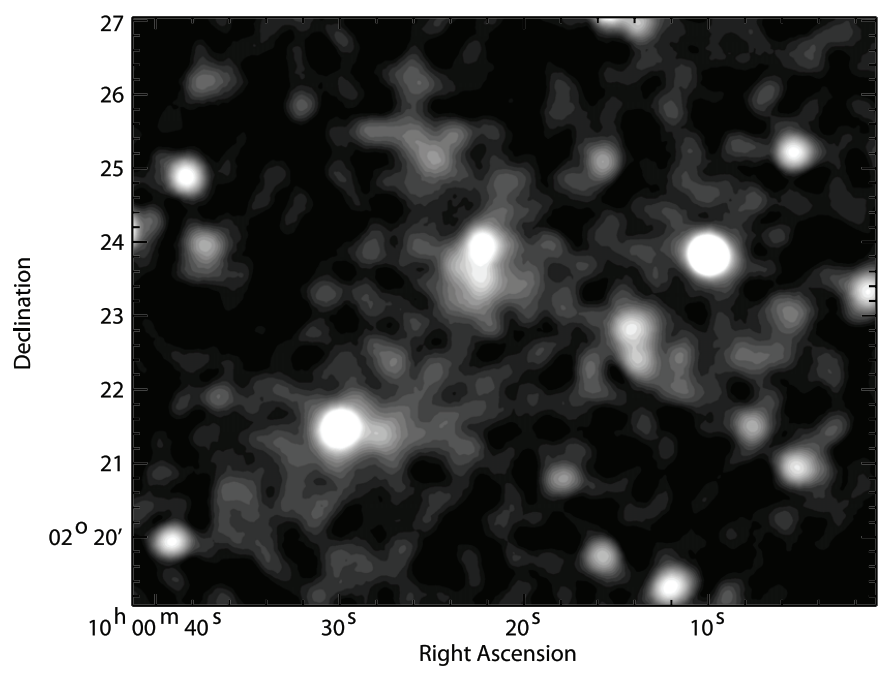

$N_{\text {dof. }}$ Zones $3 \mathrm{~b}, 3 \mathrm{c}, 4 \mathrm{~b}$, and $4 \mathrm{c}$ turned out to be nonthermal in origin, likely AGNs, while zone 5 corresponds to a bright foreground star. A power-law fit with a photon index of $\Gamma=2.3 \pm$ 0.1 to zone $1 \mathrm{c}$ gives a better $\chi^{2}$ value $\left(\chi^{2}=1.2\right.$, compared to $\chi^{2}=2.07$ for the thermal model), which indicates that a more plausible interpretation of this zone may be a background AGN, as suggested by Brusa et al. (2007). They associate the optical counterpart of this X-ray peak with a background $\left(z_{\text {photo }}=0.89\right)$ galaxy. The galaxy has a morphology of a spiral galaxy, clearly resolved in the HST ACS image. Nevertheless, we expect the X-ray centroid of the CWAT-01 parent cluster to be located in the same area (i.e., associated with zone 1c). The overall structure of the diffuse X-ray emission suggests that the center is approximately in zone 1c. Furthermore, the center of mass calculated using stellar masses of the "high-density" galaxies (see $\S 4.2$ for details) is offset from the X-ray peak by $\sim 22^{\prime \prime}$ and is still within zone 1c. Thus, for simplicity we take the X-ray peak as the center of the CWAT-01 parent cluster for calculations in this paper, arguing that it is not far from where we would expect the cluster center to be. In Table 4 we also present the estimated properties of zone 1c, assuming its thermal origin. The properties of this zone do not deviate strongly from the expectations of cluster X-ray emission. In addition, we emphasize that the mean values of the threedimensional properties based on the spectral analysis results change only within $\sim 10 \%$ when taking CWAT- 01 as the cluster center and are consistent within the errors with the properties calculated taking the X-ray peak as the center.

The temperature of each of the four clusters (see Table 4) is consistent with the temperature range typical for poor clusters (1$3 \mathrm{keV}$; Finoguenov et al. 2001a). Following Henry et al. (2004) and Mahdavi et al. (2005), we estimate the cluster volume corresponding to the spectral extraction zones. The derived values for gas mass, gas density, entropy, and pressure are listed in Table 5.

Using the luminosity-weighted temperatures of each of the four clusters (excluding zone 1c), we estimate the total mass within the corresponding radius of the enclosed matter overdensity of 500 times the critical density $\left(M_{500}^{\text {tot }}, r_{500}\right.$ in Table 5$)$, using the $M-T$ relation from Finoguenov et al. (2001b). It is possible that the masses are up to $20 \%$ higher than the quoted values, according to recent XMM-Newton and Chandra X-Ray Observatory results on the $M$ - $T$ relation (Arnaud et al. 2005; Vikhlinin et al. 2005). The uncertainty in the total mass estimate is primarily driven by the uncertainty in the measured temperature and is found to be on the level of $40 \%$ for the reported values. In Figure 7 we compare the derived properties of the X-ray emission of the four clusters with the expectations based on local sample studies that we scale to the redshift of our cluster according to the expected evolution of shock heating (see Finoguenov et al. 2005 for details). The derived properties agree well with the prediction, which for shallow survey-type data, such as ours, is reassuring in that the identification, background subtraction, and point-source confusion issues have been properly addressed.

\subsubsection{The CWAT-01 Parent Cluster}

The spectral properties of the CWAT-01 parent cluster are extracted from zones 1a, 1b, and 1c (Fig. 6, bottom) as described in $\S$ 4.1.1. The luminosity-weighted temperature of the CWAT-01 parent cluster (excluding zone $1 \mathrm{c}$ ) is $\sim 1.7 \mathrm{keV}$ and the total mass $\sim 5.8 \times 10^{13} M_{\odot}$, which makes it consistent with being a poor cluster (Finoguenov et al. 2001a). The spatial distribution of the diffuse X-ray emission of the cluster seems to be elongated and irregular. In order to obtain an estimate of its spatial characteristics (i.e., the core radius, $r_{c}$, and the $\beta$ index), we obtain a onedimensional surface brightness profile using the $0.5-2.0 \mathrm{keV}$ 
TABLE 3

Description of the Four X-Ray-Luminous Clusters Adopted from Finoguenov et al. (2007)

\begin{tabular}{|c|c|c|c|c|c|}
\hline $\begin{array}{l}\text { Main Zone } \\
\text { (1) }\end{array}$ & $\begin{array}{c}\text { Catalog ID } \\
\text { (2) }\end{array}$ & $\begin{array}{l}\text { R.A. (J2000.0) } \\
\text { (3) }\end{array}$ & $\begin{array}{c}\text { Decl. (J2000.0) } \\
\text { (4) }\end{array}$ & $\begin{array}{c}\text { Flux } \\
\left(10^{-14} \mathrm{ergs} \mathrm{cm}^{-2} \mathrm{~s}^{-1}\right) \\
(5)\end{array}$ & $\begin{array}{c}z_{\text {photo }} \\
\text { (6) }\end{array}$ \\
\hline 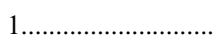 & 78 & 100028.337 & +02 2121.6 & $2.63 \pm 0.15$ & 0.22 \\
\hline ...................... & 82 & 100025.454 & +02 2519.2 & $0.76 \pm 0.07$ & 0.22 \\
\hline 3. & 85 & 100021.852 & +022342.0 & $2.44 \pm 0.15$ & 0.22 \\
\hline 4.......................... & $87^{\mathrm{a}}$ & 100013.925 & +022248.0 & $1.24 \pm 0.10$ & 0.40 \\
\hline
\end{tabular}

Notes.-We list the main zone (col. [1]; see $\S 4.1 .1$ and Fig. 6 for details) associated with the X-ray cluster catalog ID (col. [2]), the cluster's position (cols. [3] and [4]), the corresponding flux and error (col. [5]) and the photometric redshift (col. [6]). Units of right ascension are hours, minutes, and seconds, and units of declination are degrees, arcminutes, and arcseconds.

${ }^{a}$ Finoguenov et al. (2007) associated this extended X-ray emission with an optical overdensity at higher redshift. There seem to be, however, two X-ray features in their cluster catalog, one associated with this background cluster and the other with the overdensity we find at $z \sim 0.22$. (Note that the position of this cluster is not exactly coincident with the location of zone 4 a [see Fig. 6], where we find the optical overdensity at $z \sim 0.22$.) The revised cluster catalog (A. Finoguenov et al. 2007, in preparation), produced using the complete set of XMM-Newton observations, will have a separate entry for the $z \sim 0.22$ structure.

background-subtracted image corrected for exposure time. We fit the radial profile with a two-component model: (1) a Gaussian distribution to describe the emission of the inner $\sim 20^{\prime \prime}$, and (2) a traditional $\beta$-model (Cavaliere \& Fusco-Femiano 1976) for the underlying cluster. The models are centered on the main peak of the X-ray emission. A $\beta$-model with $\beta=0.57 \pm 0.06$ and $r_{c}=48.0^{\prime \prime}+8.7^{\prime \prime} .^{\prime \prime}=170.5_{-63.9}^{+30.9} \mathrm{kpc}$ is a good representation of the cluster X-ray emission. The extended cluster component yields a count rate in the (0.5-2.0) keVenergy range out to $r_{500}$ of $4.5 \times 10^{-3}$ count s$^{-1}$. We find that the luminosity of the cluster that hosts CWAT- 01 is $3.6 \times 10^{42} \mathrm{ergs} \mathrm{s}^{-1}$, consistent with $L-T$ relation of Markevitch (1998), Mulchaey (2000), and Osmond \& Ponman (2004).

Using the above derived values of $\beta$ and $r_{c}$ and a temperature of $k T \sim 2.26 \mathrm{keV}$ (zone 1b), we compute the central number density $\left(n_{0}\right)$ as described in Sakelliou et al. (1996). The central number density corresponds to $n_{0}=1.085_{-0.08}^{+1.12} \times 10^{-3} \mathrm{~cm}^{-3}$, which is in agreement with the result of the spectral analysis (Table 5). In $\S 5.2$ we use the derived quantities (i.e., $r_{c}$ and $\beta$ ) for hydrodynamic models explaining the bending of the jets of CWAT-01 and constraining the velocity of the host galaxy relative to the ICM.

TABLE 4

Results from the X-Ray Spectral Analysis

\begin{tabular}{|c|c|c|c|c|}
\hline $\begin{array}{l}\text { Zone } \\
(1)\end{array}$ & $\begin{array}{c}k T \\
(\mathrm{keV}) \\
(2)\end{array}$ & $\begin{array}{c}\text { Normalization }\left(\times 10^{-5}\right) \\
\text { (3) }\end{array}$ & $\begin{array}{l}\chi_{r}^{2} \\
(4)\end{array}$ & $\begin{array}{c}N_{\text {dof }} \\
(5)\end{array}$ \\
\hline 1a.......................... & $1.08 \pm 0.26$ & $5.4 \pm 2.9$ & 1.06 & 33 \\
\hline 1b.......................... & $2.26 \pm 0.74$ & $3.5 \pm 1.4$ & 1.12 & 19 \\
\hline $1 \mathrm{c}$ & $2.37 \pm 0.45$ & $4.1 \pm 1.1$ & 2.07 & 16 \\
\hline 2 & $1.40 \pm 0.45$ & $2.0 \pm 1.2$ & 1.38 & 16 \\
\hline 3а........................ & $1.46 \pm 0.31$ & $4.5 \pm 2.2$ & 1.18 & 31 \\
\hline .................. & $\cdots$ & $\cdots$ & $\ldots$ & $\cdots$ \\
\hline $3 c^{a}$. & $\ldots$ & $\ldots$ & $\ldots$ & $\ldots$ \\
\hline ………...... & $1.47 \pm 0.61$ & $2.2 \pm 0.8$ & 0.98 & 19 \\
\hline $4 b^{\mathrm{a}}$. & $\ldots$ & $\ldots$ & $\ldots$ & $\cdots$ \\
\hline $4 c^{a}$. & $\cdots$ & . & $\cdots$ & $\cdots$ \\
\hline $5^{\mathrm{b}}$ & $\cdots$ & $\cdots$ & $\ldots$ & . \\
\hline
\end{tabular}

Notes.-Col. (1): Spectral extraction zone (see Fig. 6 for reference) Col. (2): Temperature. Col. (3): Normalization. Col. (4): $\chi^{2}$ value. Col. (5): Number of degrees of freedom, $N_{\text {dof }}$.

a Nonthermal in origin.

b Foreground star.

\subsection{Optical Properties}

\subsubsection{Cluster Identification Using Overdensities: Voronoi Tesselations}

To map the galaxy overdensity in the area of the X-ray cluster assembly we use the Voronoi tessellation-based approach (VTA; e.g., Ramella et al. 2001; Kim et al. 2002; Botzler et al. 2006). The VTA has several advantages over other overdensity estimators that make it favorable for the scope of this paper. First, no a priori assumptions about cluster properties (e.g., density profile) are necessary, making the technique sensitive to elongated, i.e., nonsymmetrical, structures (Botzler et al. 2006). Second, we are mainly interested in substructure that can efficiently be revealed with the VTA.

A "Voronoi tessellation" on a two-dimensional distribution of points (called nuclei) is a subdivision of the plane into polygonal regions (nuclei lying on the edge may have open polygons), where each of them contains one and only one nucleus. Each region consists of the set of points in the plane that are closer to that nucleus than to any other. The algorithm used here for the construction of the Voronoi tessellation and calculation of the local densities is the varea code written by Botzler et al. (2006), which encompasses the triangle code by Shewchuk (1996). Our goal is to quantify the clustering in the area where CWAT-01 is located; thus, the input for the Voronoi tessellation (nuclei) are galaxy positions drawn from the COSMOS photometric redshift catalog (Capak et al. 2007; Mobasher et al. 2007). The VTA then defines the effective area, $A$, that a galaxy occupies in the two-dimensional space. Taking the inverse of the effective area gives the local density of the galaxy, $\rho_{\text {local }}=1 / A$.

The selection criteria we apply to the COSMOS photometric redshift catalog are the following: we select objects classified as galaxies (STAR $<1$ ) in the redshift bin of width $\Delta z=0.2$ centered at the CWAT-01 host galaxy's redshift reported in the catalog. The mean $2 \sigma$ error of the selected galaxies is $0.11 \pm 0.06$ in photometric redshifts. Thus, $67 \%$ of our galaxies have $2 \sigma$ errors in photometric redshifts better than 0.17 and $95 \%$ better than 0.23 .

To robustly estimate the background density, we apply the VTA to a region $\sim 10$ times larger than the region of interest, which is $10^{\prime} \times 8^{\prime}$, corresponding to $\sim(2.1 \times 1.7) \mathrm{Mpc}^{2}(z=0.22)$. In addition, we run Monte Carlo simulations by randomly redistributing the total number of galaxies in the analyzed field. Then we apply the VTA to each generated field and calculate the mean density, resulting in a distribution of background densities with the corresponding standard deviation, $\overline{\rho_{\text {bkg }}} \pm \sigma_{\text {bkg }}$. We define overdense 
TABLE 5

X-Ray Properties of the Clusters

\begin{tabular}{|c|c|c|c|c|c|c|c|c|}
\hline $\begin{array}{c}\text { Zone } \\
\text { (1) }\end{array}$ & $\begin{array}{c}M_{\text {gas }} \\
\left(10^{11} M_{\odot}\right) \\
(2)\end{array}$ & $\begin{array}{c}n_{e} \\
\left(10^{-4} \mathrm{~cm}^{-3}\right) \\
(3)\end{array}$ & $\begin{array}{c}S \\
\left(\mathrm{keV} \mathrm{cm}^{2}\right) \\
(4)\end{array}$ & $\begin{array}{c}p \\
\left(10^{-13}{\left.\text { dyn } \mathrm{cm}^{-2}\right)}_{(5)}\right.\end{array}$ & $\begin{array}{c}r_{\min } \\
(\mathrm{Mpc}) \\
(6)\end{array}$ & $\begin{array}{c}r_{\max } \\
(\mathrm{Mpc}) \\
(7)\end{array}$ & $\begin{array}{c}r_{500} \\
(\mathrm{Mpc}) \\
(8)\end{array}$ & $\begin{array}{c}M_{500}^{\text {tot }} \\
\left(10^{13} M_{\odot}\right) \\
(9)\end{array}$ \\
\hline 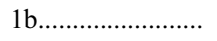 & $5.9 \pm 1.1$ & $6.7 \pm 1.2$ & $295.6 \pm 103.4$ & $24.0 \pm 9.0$ & 0.060 & 0.315 & 0.490 & 5.8 \\
\hline $1 \mathrm{c}$ & $2.00 \pm 0.25$ & $23.7 \pm 3.0$ & $133.4 \pm 27.5$ & $90.0 \pm 20.0$ & 0.000 & 0.115 & $\ldots$ & $\ldots$ \\
\hline 2 & $2.7 \pm 0.7$ & $8.3 \pm 2.3$ & $158.4 \pm 58.7$ & $18.8 \pm 7.9$ & 0.000 & 0.183 & 0.438 & 4.1 \\
\hline 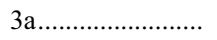 & $8.5 \pm 1.9$ & $6.0 \pm 1.3$ & $204.2 \pm 52.3$ & $1.4 \pm 0.4$ & 0.071 & 0.253 & 0.449 & 4.4 \\
\hline
\end{tabular}

Notes._Col. (1): Zones used for the spectral analysis (see Fig. 6). Col. (2): Gas mass. Col. (3): Gas density. Col. (4): Entropy. Col. (5): Pressure. Col. (6): Minimum radius of the extraction zone. Col. (7): Maximum radius of the extraction zone. Col. (8): Radius corresponding to matter overdensity of 500 times the critical density, $r_{500}$. Col. (9): Total mass within $r_{500}$.

regions as regions that have local density values of $\rho_{\text {local }}>$ $\overline{\rho_{\text {bkg }}}+10 \sigma_{\text {bkg }}$.

Using the COSMOS Subaru $g^{+}$and $r^{+}$bands, we show in Figure 8 the $g^{+}-r^{+}$versus $r^{+}$color-magnitude diagram (CMD) of galaxies with local density values $\rho_{\text {local }}>\overline{\rho_{\mathrm{bkg}}}+10 \sigma_{\mathrm{bkg}}$ and within the $\sim(2.1 \times 1.7) \mathrm{Mpc}^{2}$ area encompassing the cluster assembly. Galaxies in masked-out regions (around saturated objects) are excluded to reduce the number of artifacts (note that excluding the masked-out galaxies from the input sample for the VTA would only slightly lower the mean background density value). We also impose a magnitude cut of $B_{j}<26.5$ and $g^{+}<$ 26.5 to exclude noise artifacts that are presumably due to the $g^{+}$ and $B_{j}$ detection limits for extended sources. We therefore define the final sample of "high-density" galaxies as galaxies that satisfy the following criteria: (1) $\rho_{\mathrm{local}}>\overline{\rho_{\mathrm{bkg}}}+10 \sigma_{\mathrm{bkg}}$, (2) $B_{j}<$ 26.5 and $g^{+}<26.5$, and (3) the galaxies are outside masked-out regions around saturated objects.

\subsubsection{Cluster Assembly Structure}

The Voronoi tessellation for the $\sim(2.1 \times 1.7) \mathrm{Mpc}^{2}$ area surrounding the cluster assembly with indicated high-density galaxies is shown in Figure 9. The large-scale overdensity is elongated in northwest-southeast direction with several obvious subclumps. The spatial distribution of the galaxies seems not to be spherically symmetric, but irregular, both on large and small scales. In the areas around saturated stars (i.e., masked-out regions) we lose all information about clustering.

In Figure 10 the high-density galaxies are overlaid on the Subaru $i^{+}$-band image. For comparison, diffuse X-ray-emission contours are also shown. It is evident that the high-density galaxies display a complex and irregular spatial distribution, consistent with the irregular and elongated distribution of the diffuse X-ray emission. Each X-ray-identified poor cluster has a counterpart in optical overdensities approximately following the distribution of the X-ray emission. Note that the X-ray cluster corresponding to zone $4 \mathrm{a}$ is associated with an optical overdensity with a mean redshift of $z \approx 0.22$ like the other clusters. This is additionally confirmed by the SDSS J10006.65+022225.98 galaxy spectrum (see Table 2 for details).

The optical overdensities reveal, in addition, a clustering region northwest from the diffuse X-ray emission (LG-N in Fig. 10) not detected in the $0.5-2.0 \mathrm{keV}$ X-ray band. The SDSS J10004.35+ 022550.71 galaxy spectrum confirms that this structure is at the
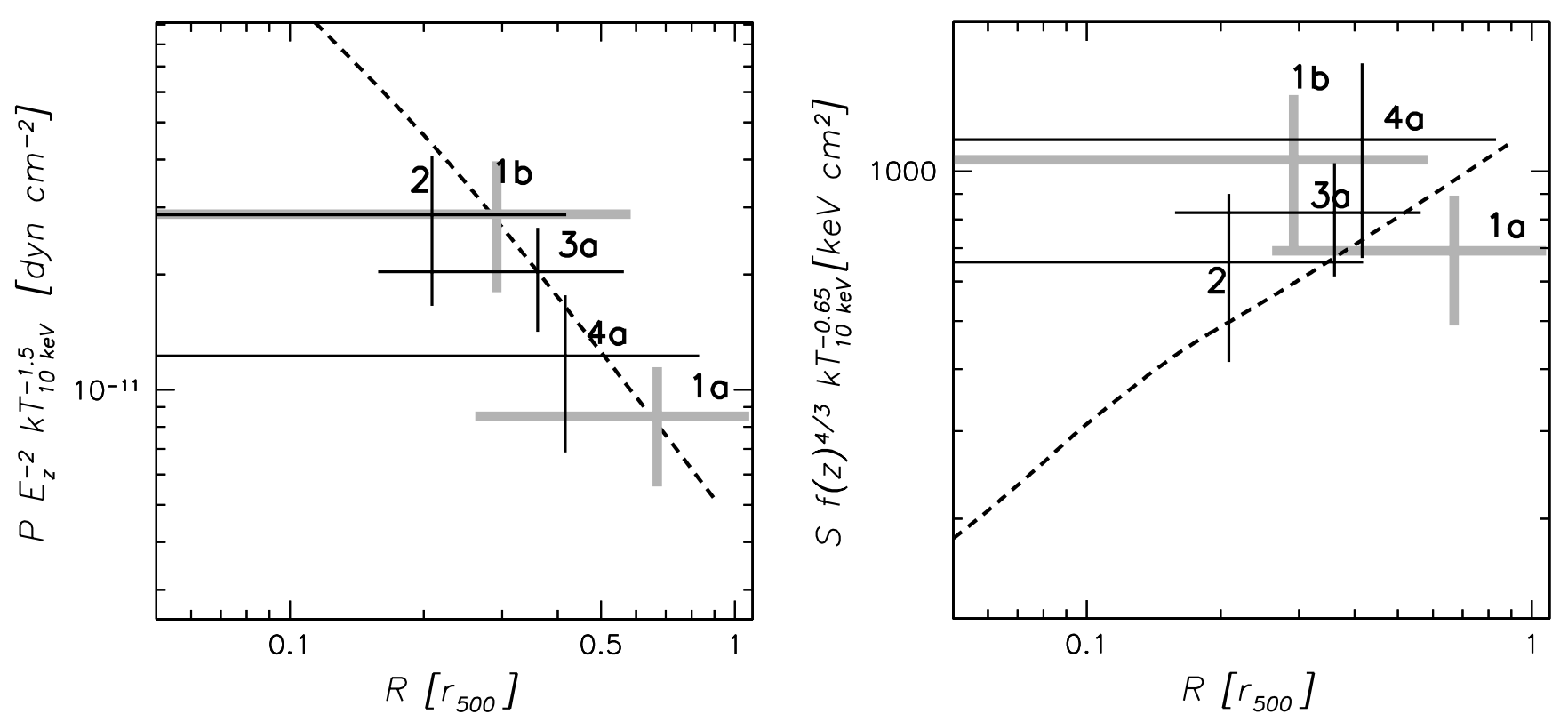

FIG. 7.-Pressure (left) and entropy (right) as a function of cluster radius for different spectral extraction zones (labeled in each panel; for comparison see Fig. 6). Thick crosses (zones 1a and 1b) represent the CWAT-01 parent cluster. The length of the crosses indicates the $1 \sigma$ errors. The dashed lines show the expected pressure and entropy behavior based on local cluster studies and scaled to the redshift of this system (see text for details). 


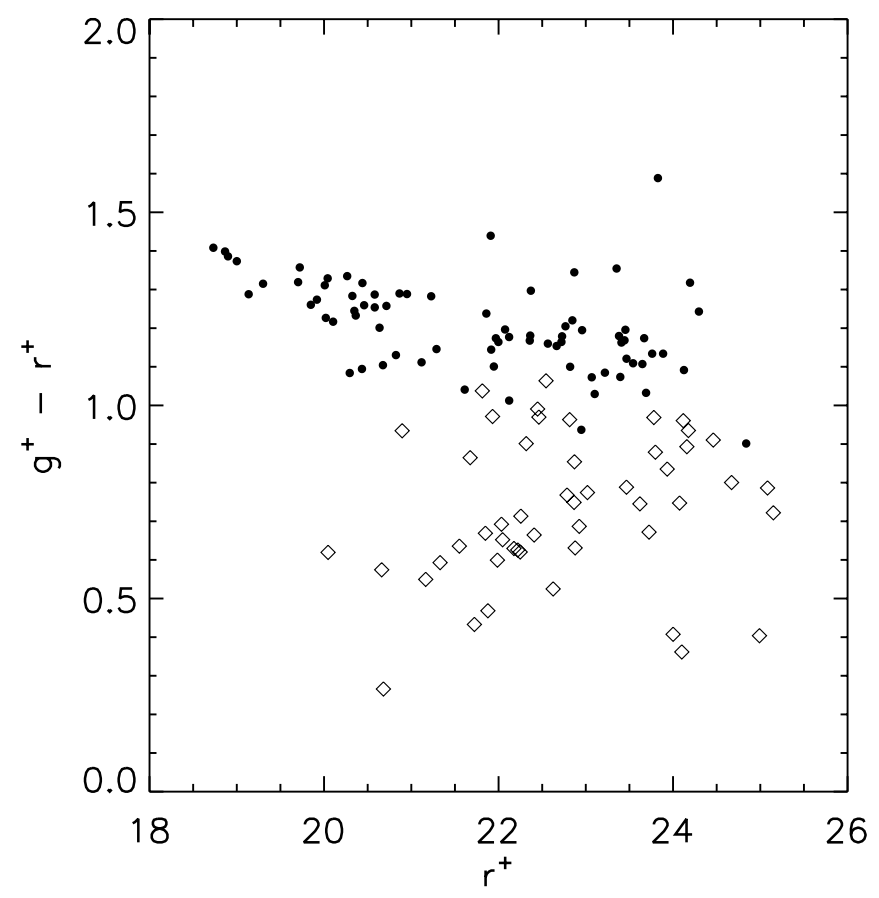

FIG. 8. - The $g^{+}-r^{+}$vs. $r^{+}$CMD using the COSMOS Subaru $g^{+}$and $r^{+}$bands. The galaxies shown in the CMD are galaxies $\left(g^{+}<26.5\right.$ and $\left.B_{j}<26.5\right)$ within the cluster area of interest, corresponding to $\sim(2.1 \times 1.7) \mathrm{Mpc}^{2}(z=0.22)$, which satisfy the overdensity criterion imposed in the VTA analysis: $\rho_{\text {local }}>\overline{\rho_{\mathrm{bkg}}}+10 \sigma_{\mathrm{bkg}}$ (see $\S$ 4.2.1 for details). Galaxies in masked-out regions (around saturated objects) are excluded. Filled symbols represent early-type galaxies (SED type $<2.5$ ), while open symbols stand for late-type galaxies (SED type $>2.5$ ).

same redshift as the whole cluster system. We assume that LG-N is a loose group bound to the cluster assembly.

\subsubsection{Substructure in the CWAT-01 Parent Cluster}

The spatial distribution of the high-density galaxies (Fig. 11, top) in the CWAT-01 parent cluster is irregular and elongated with two dominant subclumps: (1) a western overdensity (including the CWAT-01 host galaxy) extended in the northwestsoutheast direction ( $\mathrm{SC1}$ in Fig. 11) and (2) an eastern overdensity elongated in northeast-southwest direction (SC2). There are three bright foreground stars contaminating the CWAT-01 parent cluster area. Nevertheless, those masked-out regions should not affect our results substantially since they are located at the outer parts of the parent cluster. Contrary to the expectation in relaxed systems where one would expect early-type galaxies to be centrally concentrated around the bottom of the cluster potential well, the distribution of the early-type galaxies (SED type $<2.5$ ) in the CWAT-01 parent cluster is spatially elongated and coincident with subclumps $\mathrm{SC} 1$ and $\mathrm{SC} 2$ (see Fig. 11). Late-type galaxies are preferentially at the outskirts of the cluster. The brightest galaxy in the cluster is the CWAT-01 host galaxy $\left(r^{+}=18.899 \pm\right.$ $0.004, M_{V}=-22.9 \pm 0.1$ ). This is not surprising, since previous studies that have shown that WATs are generally associated with BCGs (e.g., Burns 1981). In addition, the brightest galaxies $\left(M_{V}<-20.5\right)$ in the cluster are strongly clustered in the region around CWAT-01, while only one (i.e., the second brightest in absolute $V$ magnitude) is located at the outskirts of the cluster (see Fig. 11, top).

Using the stellar masses reported in the COSMOS photometric redshift catalog (Mobasher et al. 2007) of the high-density galaxies, we compute the position of the center of mass (indicated in Fig. 11, bottom). The offset of the center of mass from the main peak in the diffuse X-ray emission is only $\sim 22^{\prime \prime}$. Note that because of the bias introduced by the masked-out regions in the cluster, the center of mass may be closer to the main X-ray peak than given above.

\section{DISCUSSION}

The unified theory for the mechanism responsible for bending the jets of WAT radio galaxies is the dynamic pressure exerted on the jets by the ICM due to the relative motion between the galaxy and the ICM. In $\S 5.1$ we compare the minimum pressure present in the radio jets to the thermal ICM pressure in order to investigate the confinement of the jets. We have shown in previous sections that CWAT-01 is associated with the BCG in its parent cluster. Therefore, it is expected to be at rest in the minimum of the gravitational potential (e.g., Bird 1994). In order to constrain the relative velocity between CWAT-01's host galaxy and the ICM we apply several hydrodynamic models explaining the bending of the jets of WATs (§5.2). In $\S 5.3$ we suggest possible merger and encounter scenarios responsible for the bending of the jets. The environment of CWAT-01 on larger scales (i.e., the cluster assembly) is discussed in $\S 5.4$.

\subsection{Pressure Balance}

It is often assumed that the radio jets are confined by the ICM (e.g., Miley 1980 and references therein); thus, it is interesting to compare the minimum internal pressure in the radio jets with the thermal ICM pressure. The minimum internal pressure in the radio jets was calculated in the middle part of the eastern tail (§ 3.1.4), $p_{\min }^{\text {jet }} \cong 4.3 \times 10^{-13} \mathrm{dyn} \mathrm{cm}^{-2}$, and it is lower than the ICM pressure in zone $1 \mathrm{~b}$, which contains CWAT-01, $p_{\mathrm{ICM}}^{\text {zone } 1 \mathrm{~b}}=(24 \pm 9) \times$ $10^{-13}$ dyn $\mathrm{cm}^{-2}$ (see $\S 4.1$ for details). Such a pressure imbalance is not unusual for WATs (e.g., Hardcastle \& Sakelliou 2004). It implies either some departure from the minimum energy condition (which is almost equal to equipartition between the relativistic particles and the magnetic fields in the jets) or a contribution to the pressure from particles that do not participate in equipartition such as thermal protons. However, one should be careful in comparing the two pressure values, due to the low resolution of the X-ray data and the numerous assumptions in the minimum-pressure calculation. Nevertheless, the ram pressure models we apply to CWAT-01 in order to constrain the relative velocity between the galaxy and the ICM $(\S 5.2)$ should not be affected by this pressure imbalance. The inherent assumption in these models is that the dynamic ram pressure of the ICM is comparable with the pressure difference across the jet.

\subsection{Bending of the Radio Jets of CWAT-01: Constraints on the Galaxy Velocity}

In this section we apply several hydrodynamic models explaining the bending of the jets of CWAT-01 in order to constrain the velocity of CWAT-01's host galaxy relative to the ICM. The classical Euler equation describes the jets if the bulk plasma velocity in the jets is characterized by nonrelativistic motions (e.g., Jaffe \& Perola 1973; Begelman et al. 1979; Christiansen et al. 1981; Sakelliou et al. 1996). Sakelliou et al. (1996) developed a simple hydrodynamic model to describe the bending of the jets of $3 \mathrm{C} 34.16$. They assume that the forces acting on the jets are ram pressure and buoyancy, and they model the jets in the plane of the sky assuming a steady plasma flow (Sakelliou et al. 1996, their eqs. [8]-[11]). The strength of this model is that it provides a constraint on the galaxy velocity relative to the ICM solely dependent on the jet density at the point where ram pressure and buoyancy balance. At the turnover point the bending of the jet changes its direction. At this point the forces of ram pressure and buoyancy in the direction normal to the jet balance and the only 


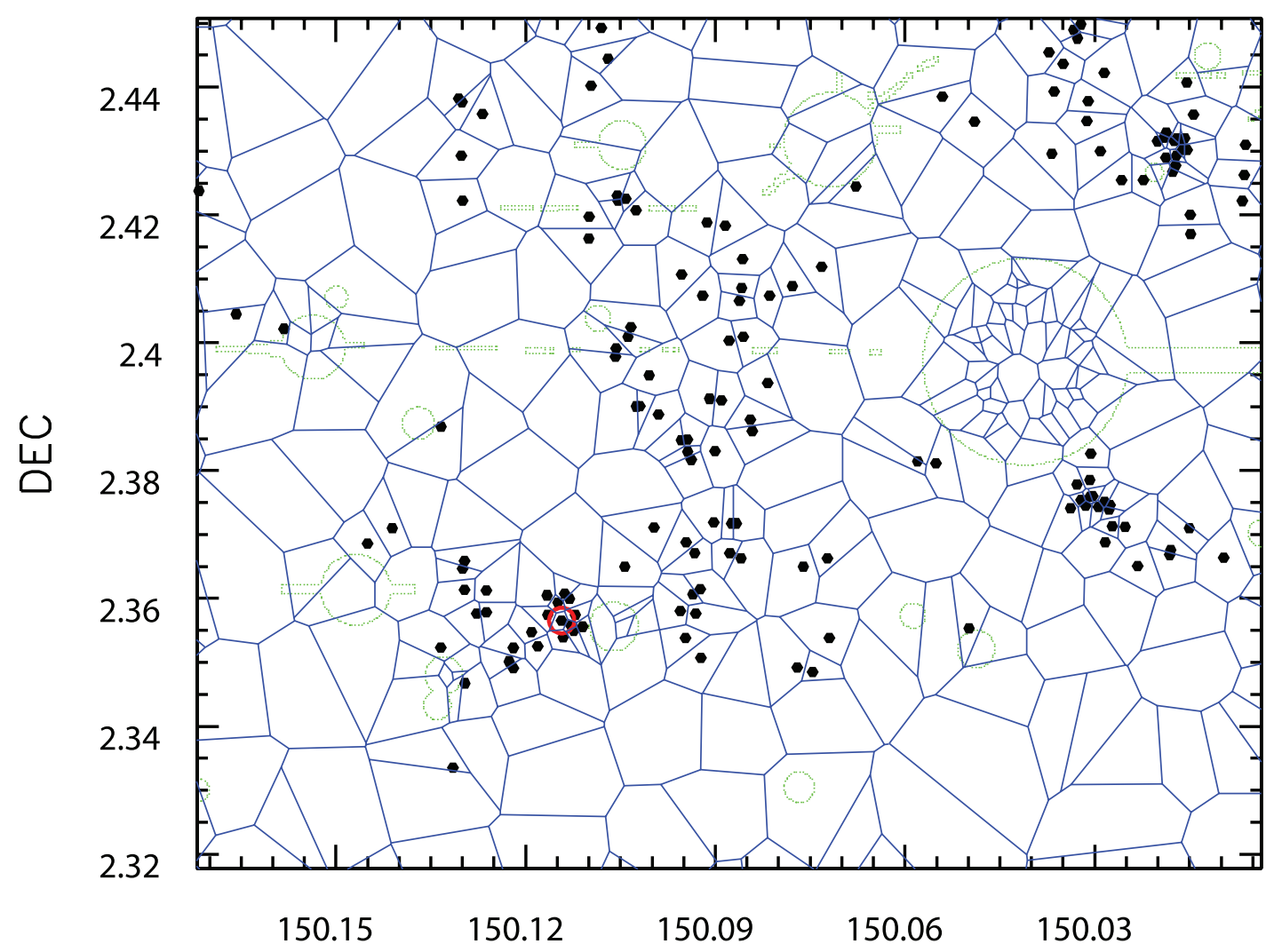

RA

FIG. 9.-VTA in the area of the cluster assembly (solid blue lines). The shown field is $\sim(2.1 \times 1.7) \mathrm{Mpc}^{2}($ at $z=0.22)$ in size and $\approx 10$ times smaller than the total area analyzed. Masked-out regions (around saturated objects) in the photometric redshift catalog are marked with dotted green lines (see text for details). The points represent highdensity galaxies that (1) satisfy our overdensity criterion of $\rho_{\text {local }}>\overline{\rho_{\mathrm{bkg}}}+10 \sigma_{\mathrm{bkg}}$, (2) satisfy the magnitude criterion of $g^{+}<26.5$ and $B_{j}<26.5$, and (3) are not located in masked-out regions. CWAT-01 is marked with the open circle with solid edges.

unknowns are the galaxy velocity and the density of the jet at this point:

$$
v_{\text {gal }}^{2}=\frac{3 \beta k T_{\mathrm{ICM}} h}{\mu m_{p} r_{c}} \frac{r_{\mathrm{to}} / r_{c}}{1+\left(r_{\mathrm{to}} / r_{c}\right)^{2}} \frac{\hat{\boldsymbol{r}} \cdot \hat{\boldsymbol{n}}}{\hat{\boldsymbol{v}}_{\mathrm{gal}} \cdot \hat{\boldsymbol{n}}}\left(1-\frac{\rho_{j}}{\rho_{\mathrm{ICM}}}\right) .
$$

Here $r_{\text {to }}$ is the radial distance from the cluster center to the turnover point (projected on the plane of the sky), $r_{c}$ and $\beta$ are the core radius and the standard hydrostatic-isothermal $\beta$ model parameter, respectively (see $\S 4.1 .2$ ), $\hat{\boldsymbol{n}}$ is the normal vector to the jet in the plane of the sky, $v_{\text {gal }}$ is the component of the galaxy velocity in the plane of the sky, $h$ is the scale height of the jet, $\rho_{j}$ and $\rho_{\mathrm{ICM}}$ are the jet and ICM densities, respectively, $k T_{\mathrm{ICM}}$ is the ICM temperature in $\mathrm{keV}(\sim 2.26 \mathrm{keV}), \mu$ is the mean molecular weight, and $m_{p}$ is the proton mass.

The $1.4 \mathrm{GHz}$ radio map (Fig. 1) clearly shows that the radio jets bend twice. Near the optical counterpart the jets turn to the south. The second bend of the jets is toward the southwest direction (points E1 and W1 in Fig. 1). The first bend can be attributed to ram pressure as a result of the relative motion of the galaxy through the ICM. At larger radii buoyancy takes over and the jets are pushed toward lower density regions in the ICM. Figure 12 shows the galaxy velocity as a function of the ratio of the jet density to the ICM density, $\rho_{j} / \rho_{\mathrm{ICM}}$, calculated at the point $\mathrm{W} 1$ (thick solid line). The allowed range in velocities is indicated (broad hatched region). The limiting velocity of the galaxy relative to the
ICM (in the limit $\rho_{j} / \rho_{\mathrm{ICM}} \rightarrow 0$ ) is $v_{\text {gal }} \sim 400_{-110}^{+150} \mathrm{~km} \mathrm{~s}^{-1}$. Note that $v_{\text {gal }}$ measures the projected velocity on the plane of the sky; thus, any inclination of CWAT-01 to the line of sight would result in an additional line-of-sight component of velocity, thus increasing the total speed of the system. The upper and lower limits of the galaxy velocity were computed taking into account the errors of $r_{c}, \beta$, and $k T$. Additional uncertainties are introduced through the estimate of $\hat{\boldsymbol{n}}$ at the turnover point and $\hat{\boldsymbol{r}} \cdot \hat{\boldsymbol{n}}$, which depends on the position of the center of the total mass of the cluster (discussed in $\S 4.1 .1$ ). Thus, the derived velocity is a rough estimate of CWAT-01's velocity relative to the ICM as a function of $\rho_{j} / \rho_{\mathrm{ICM}}$.

Another hydrodynamic model, which we apply to CWAT-01 to estimate the galaxy velocity, was first proposed by Begelman et al. (1979) to explain the jets in NGC 1265. The curvature of the jets is again assumed to be produced by ram pressure exerted on the galaxy as it moves through the ICM. The ram pressure is balanced by the centrifugal force exerted by the jet as it curves:

$$
\frac{\rho_{\mathrm{ICM}} v_{\mathrm{gal}}^{2}}{h}=\frac{\rho_{j} v_{j}^{2}}{R},
$$

where $v_{j}$ is the bulk jet velocity, $h$ is the scale height, $R$ is the radius of curvature, $v_{\text {gal }}$ is the galaxy velocity, and $\rho_{\mathrm{ICM}}$ and $\rho_{j}$ are the ICM and jet densities, respectively. Placing the mean jet velocity of the velocity range derived in $\S 3.1 .5, v_{j} \sim 0.045 c$, into 


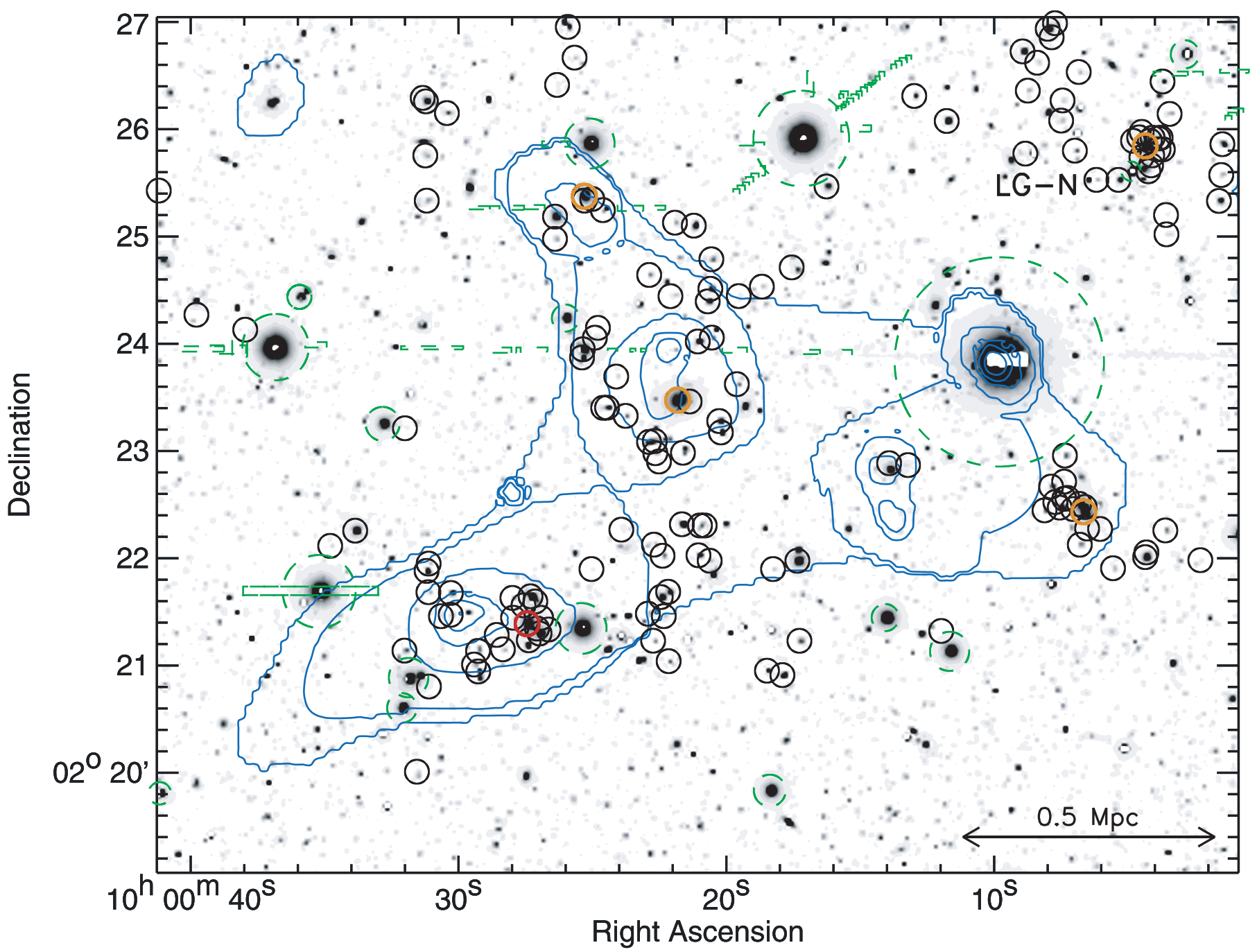

Fig. 10.-Gray-scale Subaru $i^{+}$-band image of the cluster area overlaid with X-ray contours (blue). The contour levels are the same as in Fig. 6. The shown area is $\sim(2.1 \times 1.7) \mathrm{Mpc}^{2}$ (at $\left.z=0.22\right)$ with circles (black) denoting the high-density galaxies (the same galaxies as in Fig. 9). Masked-out regions around saturated objects (drawn from the COSMOS photometric catalog) are indicated with dashed green lines. CWAT-01 is marked with the red circle with thick edges. Orange circles indicate galaxies that have spectra (see $\S 2.4$ and Tables 1 and 2 for details). LG-N labels the overdensity evident from the VTA but not detected in X-rays (see text for details). The 0.5 Mpc projected distance is indicated for reference.

equation (6), using an estimate of the scale height (at point $\mathrm{C} 0$ in Fig. 1) of $h \sim 1.7^{\prime \prime}(\sim 6 \mathrm{kpc})$ and the radius of curvature of $R \sim$ $10^{\prime \prime}(\sim 35 \mathrm{kpc})$, we show the galaxy velocity as a function of the $\rho_{j} / \rho_{\text {ICM }}$ ratio in Figure 12 (dashed lines). Indicated is the galaxy velocity range corresponding to the jet velocity range, $0.04 c \lesssim$ $v_{j} \lesssim 0.05 c$ (narrow hatched region).

Requiring that the conditions of both equations, equations (5) and (6), are satisfied (as illustrated in Fig. 12), we obtain an estimate of both the galaxy velocity, $v_{\text {gal }}$, and the jet density, $\rho_{j} ; v_{\text {gal }}$ is in the range of about $400_{-100}^{+150} \mathrm{~km} \mathrm{~s}^{-1}$, and $\rho_{j}$ is $0.005_{-0.003}^{+0.01} \rho_{\mathrm{ICM}}$. The ICM density is known from the spectral analysis, $\rho_{\text {ICM }}=$ $(6.94 \pm 1.27) \times 10^{-4} \mathrm{~cm}^{-3}$; thus, the estimated jet density with the corresponding errors is about $0.03_{-0.02}^{+0.09} \times 10^{-4} \mathrm{~cm}^{-3}$.

So far we have neglected possible in situ particle acceleration within the jets. If particle reacceleration occurs, then the bulk lifetime of the synchrotron electrons in the jets would be higher than the 13 Myr estimated in $\S 3.1 .5$. A lower limit of the galaxy velocity can then be estimated by assuming efficient conversion of kinetic energy into internal energy in the plasma jet flow (e.g., Eilek 1979). If the observed luminosity, $L$, of the jets is supplied by conversion of the bulk kinetic energy with an efficiency, $\epsilon$, then $L \sim(\pi / 2) \rho_{j} h^{2} v_{j}^{3} \epsilon$ (e.g., Burns 1981; O’Donoghue et al. 1993). Substituting $v_{j}$ in terms of the luminosity into equation (6), one gets

$$
v_{g} \gtrsim\left(\frac{2 L}{\pi}\right)^{1 / 3} \rho_{j}^{1 / 6} \rho_{\mathrm{ICM}}^{-1 / 2} h^{-1 / 6} R^{-1 / 2} \epsilon^{-1 / 3},
$$

which is only weakly dependent on the jet density, $\rho_{j}$. For an efficiency of $\sim 10 \%$, assuming the above derived jet density, the galaxy velocity is roughly $v_{g} \gtrsim 350 \mathrm{~km} \mathrm{~s}^{-1}$, which is consistent with the results from the previously applied models.

The models we applied to CWAT-01 in the above discussion thus suggest that the galaxy velocity relative to the ICM is in the range of about $300-550 \mathrm{~km} \mathrm{~s}^{-1}$.

\subsection{Subcluster Merging in the CWAT-01 Parent Cluster?}

Beers et al. (1995) report a median velocity dispersion of $336 \pm$ $40 \mathrm{~km} \mathrm{~s}^{-1}$ (which is in agreement with, e.g., Ramella et al. 1994 and Ledlow et al. 1996) for a sample of MKW/AWM poor 

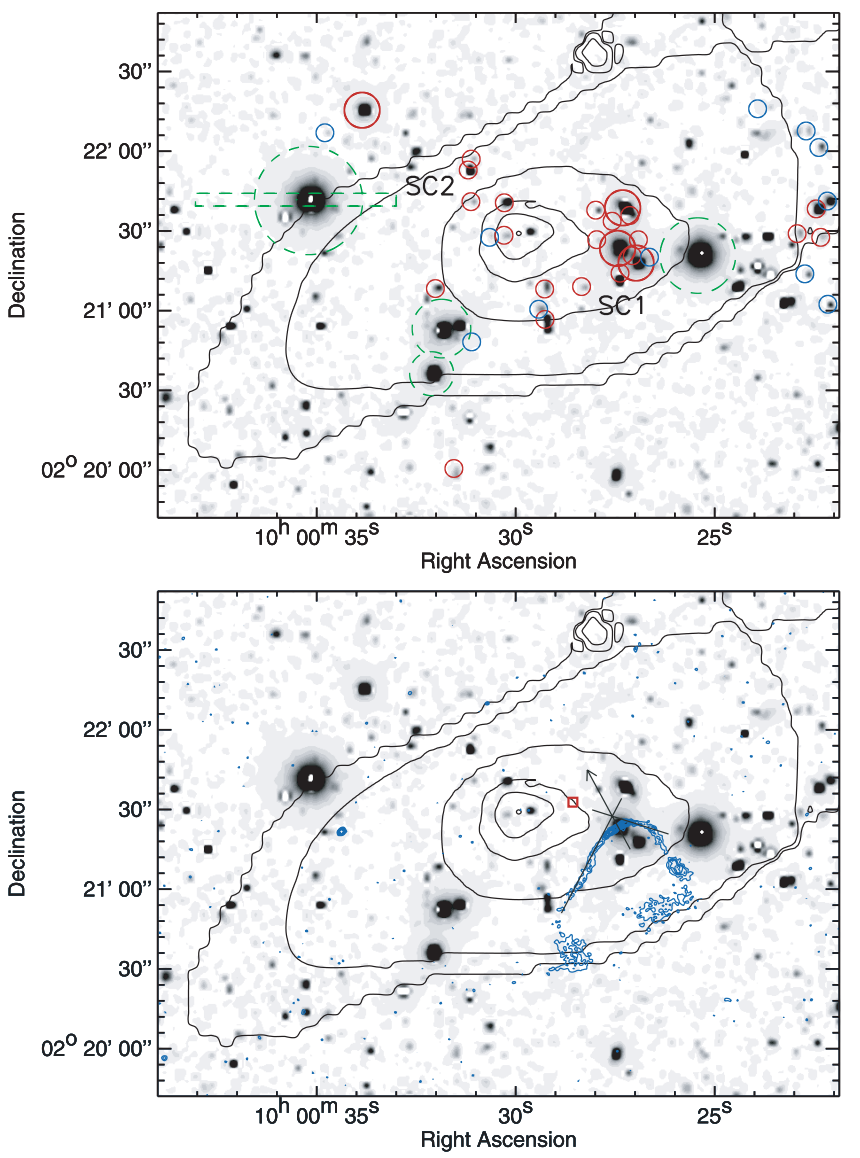

FIG. 11.-Top: Subaru $i^{+}$-band image ( gray scale) of the CWAT-01 parent cluster. Overlaid are X-ray contours with contour levels as in Fig. 6. Indicated are high-density galaxies. Red circles with solid edges denote early-type galaxies (SED type $<2.5$ ), while blue circles with solid edges indicate late-type galaxies (SED type $>2.5)$. The brightest galaxies $\left(M_{V}<-20.5\right)$ in this area are marked with red circles with thick edges. Dashed green lines mark masked-out objects. SC1 and SC2 label the two subclumps evident in the cluster (see text for details). Bottom: Subaru $i^{+}$-band image ( gray scale) and X-ray contours as in top panel overlaid with $1.4 \mathrm{GHz}$ radio contours. The radio contour levels start at the $3 \sigma$ level and increase in steps of $1 \sigma$. The bending angle of CWAT- 01 is indicated by thin lines, while the arrow indicates the velocity direction of the galaxy. The open box marks the position of the center of mass of the cluster, computed taking into account the stellar masses of the "high-density" galaxies in the CWAT-01 parent cluster (the stellar masses were drawn from the COSMOS photometric redshift catalog [Mobasher et al. 2007]).

clusters. $^{20}$ They found a velocity offset between the velocity of the central galaxy and the mean velocity of the rest of the galaxies of $\lesssim 150 \mathrm{~km} \mathrm{~s}^{-1}$ for clusters with no evidence for subclustering. The velocity range of $300-550 \mathrm{~km} \mathrm{~s}^{-1}$ we found for CWAT-01's host galaxy, which is the dominant galaxy (BCG) in its parent cluster, is significantly higher than this limit, which indicates recent merger events between less massive systems of galaxies (Bird 1994). High peculiar velocities are strongly correlated with the presence of substructure in the system (Bird 1994). Indeed, the VTA results indicate subclustering in the CWAT-01 parent cluster (SC1 and SC2 in Fig. 11). Furthermore, the brightest galaxies in the cluster are strongly concentrated around CWAT-01. We speculate that $\mathrm{SC} 1$ and $\mathrm{SC} 2$ interact and, furthermore, that $\mathrm{SC} 2$ may be infalling into the gravitational potential of SC1. This

20 A sample of 23 poor clusters of galaxies originally identified by Morgan et al. (1975) and Albert et al. (1977).

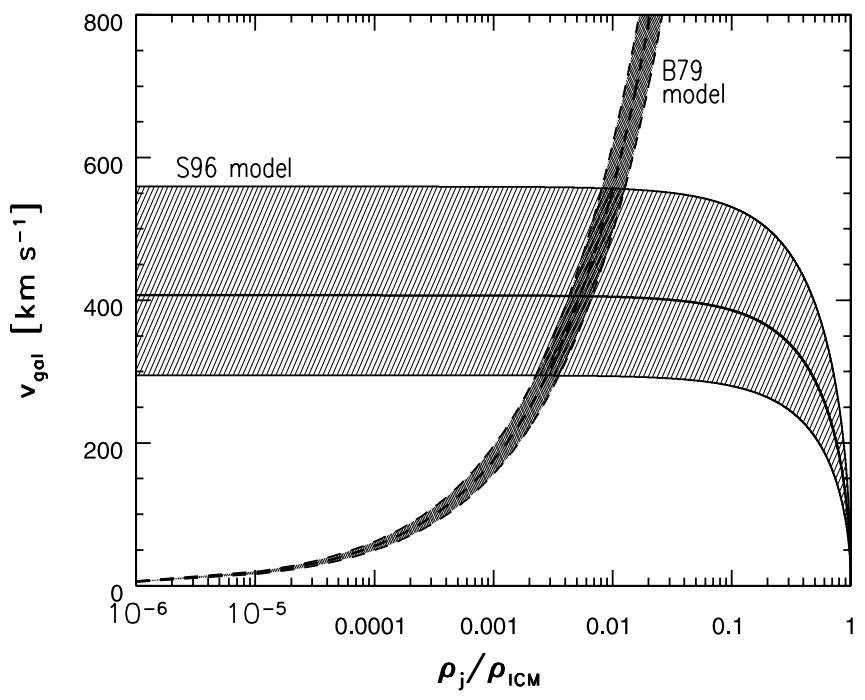

FIG. 12.-Mean galaxy velocity, $v_{\text {gal }}$ (thick lines), as a function of the ratio of the jet to ICM density, $\rho_{j} / \rho_{\mathrm{ICM}}$, with the allowed ranges (hatched regions) corresponding to our error estimates. The broad hatched region shows the galaxy velocity calculated from eq. (5) using the model developed in Sakelliou et al. (1996; S96), which takes into account ram pressure and buoyancy as forces responsible for bending the jets (see text for details). The narrow hatched region shows the galaxy velocity calculated from eq. (6) (developed by Begelman et al. 1979 ; B79) taking only ram pressure into account. If we require that the conditions of both models are satisfied, then the allowed ranges for $v_{\text {gal }}$ and $\rho_{j}$ are within the intersecting area.

interaction may cause a dynamical state of the cluster violent enough to produce the inferred relative velocity of the CWAT-01 host galaxy to the ICM needed to bend the jets in the observed way. Moreover, the irregular assembly of early-type galaxies in the CWAT-01 parent cluster (see Fig. 11) suggests that it is not a relaxed system. The elongated and irregular diffuse X-ray emission of this cluster indicates independently possible merger or accretion events in the cluster. Finoguenov et al. (2005) have shown that X-ray elongations are often seen at the outskirts of clusters and that their spectral characteristics correspond to a colder, dense gas in pressure equilibrium with the cluster. This gas is associated with accretion zones in clusters where dense parts of the filaments survive the accretion shock and penetrate the cluster outskirts.

Our results suggest that merger events within the CWAT-01 parent cluster caused such a dynamical state in the cluster, which is needed for bending the radio jets of CWAT-01 in the observed way. Such a merging scenario is consistent with conclusions of previous studies that have suggested that the bent shape of WAT galaxies is caused by mergers (e.g., Gomez et al. 1997; Sakelliou et al. 1996; Sakelliou \& Merrifield 2000).

Based on a sample of $\sim 20$ WAT galaxies that are located in Abell clusters and have ROSAT X-ray observations Sakelliou \& Merrifield (2000) have shown that WATs travel predominantly radially toward or away from the center (as defined by the X-ray centroid) of their host cluster. CWAT-01 does not seem to be on a radial motion in the projected plane of the sky, as is evident in Figure 11 (bottom). This may be a bias caused by projection effects. However, the gravitational influence of the neighboring clusters may have played a significant role in causing the inferred velocity modulus and direction of CWAT-01's host galaxy relative to the ICM.

Based on the above arguments we suggest that the radio jets of CWAT-01 were bent as a consequence of the motion of CWAT-01 

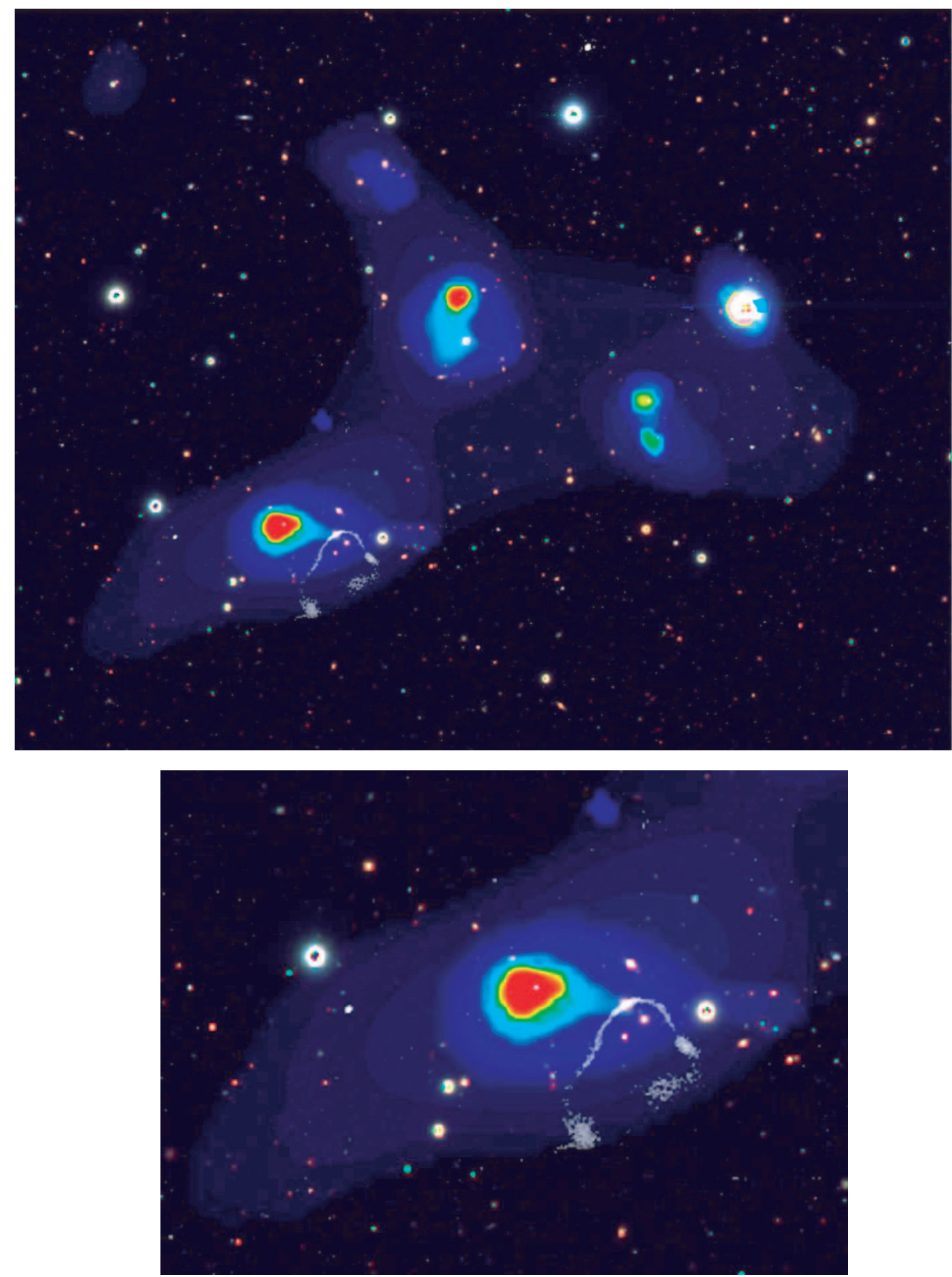

FIG. 13.-Color composite image of the cluster area (top) and the CWAT-01 parent cluster (bottom). The Subaru $B$ (blue), $V($ green $)$, and $i^{+}$(red $)$bands are displayed in the background. The diffuse X-ray emission is presented by rainbow colors, and the $1.4 \mathrm{GHz}$ map is shown in white. The top panel encompasses an area of $\sim(2.1 \times 1.7) \mathrm{Mpc}{ }^{2}$ (analogous to Fig. 10). The size of the bottom panel is as in Fig. 11.

relative to the ICM induced through interactions between subclusters (SC1 and SC2) and/or interactions between the CWAT-01 parent cluster and the other identified clusters.

\subsection{Galaxy Cluster Assembly}

Since CWAT-01 seems to be part of a very complex largescale structure, the possible gravitational influence of the other clusters on the galaxy and its immediate environment cannot be neglected. Figure 13 shows the distribution of emission from different parts of the electromagnetic spectrum within the cluster area. The diffuse X-ray emission has revealed that the CWAT-01 parent cluster is only one of the poor clusters encompassed in a larger cluster structure. The cluster assembly contains a minimum of four X-ray luminous clusters within $\sim 2 \mathrm{Mpc}$ distance. 
In addition, the VTA indicates that there is at least one more loose group (LG-N; Fig. 10) on the outskirts of the X-ray cluster assembly but not detected in the X-rays. Furthermore, the whole cluster assembly is part of a large-scale structure component (LSS 17, Scoville et al. 2007b) extended over $4 \mathrm{Mpc}$ in the north-south direction.

The direction of the jets of CWAT-01 is almost perpendicular to the X-ray elongation of the CWAT-01 parent cluster, as well as to the large-scale elongation of the X-ray emission. Although LSS 17 spatially extends $\sim 4 \mathrm{Mpc}$ in the north-south direction, the direction of its long axis (as defined in, e.g., Novikov et al. 1999 ) is in the northwest-southeast direction (see Fig. 3 in Scoville et al. 2007b). Hence, the direction of the jets of CWAT-01 is almost perpendicular also to the long axis of LSS 17. This is in disagreement with the correlation of the alignment between the direction of the jets and (1) the central X-ray emission elongation (Burns et al. 1994; Gomez et al. 1997) and (2) the supercluster axis as defined by the distribution of the nearby Abell clusters (Novikov et al. 1999). Nevertheless, the misalignment seen in CWAT-01's orientation compared to the parent cluster and to LSS 17 elongations may not be unexpected if we assume an early stage of cluster formation. Rich clusters form at the intersection of large-scale filaments. Compared to numerical simulations (e.g., Burns et al. 2002; Springel et al. 2005) of cluster evolution at $z=0.2$, the final cluster has not yet formed or relaxed at the intersection of filaments where matter is accreted from numerous filaments. Thus, in such an environment it would not be unexpected to observe a WAT galaxy with its jets not aligned with the cluster or large-scale structure elongations.

The X-ray and optical analyses indicate that each individual poor cluster within the cluster assembly is not spherically symmetric, both in the diffuse X-ray emission and in the spatial distribution of galaxies in the optical (VTA). This strongly indicates that the poor clusters are not in a dynamically relaxed state. In addition, the overall, large-scale distribution of the cluster assembly is very complex and irregular. It is likely that the loose group LG-N is bound to the system. The lack of X-ray emission from this group suggests that the system is not very massive. The flux limit for the cluster search (see $\S 2.2$ ) allows a detection of an object at $z=0.22$ with a limiting luminosity of $L_{\mathrm{X}}^{\text {limit }}=3 \times 10^{41} \mathrm{ergs} \mathrm{s}^{-1}$, which corresponds to a low-mass group (e.g., Mulchaey et al. 2003). Following the luminosity-temperature $(L-T)$ relation for groups (Osmond \& Ponman 2004) the limiting temperature then equals $T_{\mathrm{X}}^{\text {limit }} \sim 0.4 \mathrm{keV}$. Using the mass-temperature $(M-T)$ relation from Finoguenov et al. (2001b) the minimum mass of a system to be detected in the diffuse $0.5-2 \mathrm{keV}$ band at $z=0.22$ then corresponds to $M_{500}^{\text {tot,limit }} \sim 1.5 \times 10^{13} M_{\odot}$. Thus, the total mass of LG-N has to be less than this mass.

Although spectroscopic verification is needed for the physical connection of the clusters, we believe that we are witnessing a protocluster in the process of being built up of multiple galaxy clusters and groups. Adding up the estimated masses for the four clusters identified via diffuse X-ray emission (see Table 5) and the limiting mass inferred for LG-N, the resulting combined mass is $M \sim 2.0 \times 10^{14} M_{\odot}$. This is a rough estimate of the total mass of the cluster system once it is formed since it does not include the material between the clusters nor other loose groups that may be bound to the cluster assembly (the cluster assembly is only part of LSS 17). Thus, the estimated mass that the final cluster may have after the individual clusters merge would correspond to $\sim 20 \%$ of the Coma Cluster's total mass. This is the first time such a complex dynamically young cluster system in the process of formation is identified via a WAT radio galaxy.

\section{SUMMARY AND CONCLUSIONS}

We have analyzed a wide-angle tail (WAT) radio galaxy, CWAT01, first resolved in the VLA-COSMOS survey. The multiwavelength data set of the COSMOS survey has enabled us to identify and analyze the environment of CWAT-01 in several independent ways. The cluster structure revealed via CWAT-01 seems to be more complex than any structure hosting WAT galaxies reported in the past. We summarize the basic findings of this analysis as follows:

The lengths of the eastern and western radio jets of CWAT-01 are $\sim 210$ and $\sim 160 \mathrm{kpc}$, respectively, and the bending angle is $\sim 100^{\circ}$ in the projected plane of the sky. It seems to be asymmetric, and at this point we cannot rule out that the asymmetry is caused by projection effects. The $1.4 \mathrm{GHz}$ radio power of $P_{1.4}=$ $2.0 \times 10^{24} \mathrm{~W} \mathrm{~Hz}^{-1}$ puts CWAT-01 on the lower end of the FR I-II break region where WATs are usually found.

The host galaxy of CWAT-01 is an elliptical galaxy with a shallower surface brightness profile than predicted by the de Vaucouleurs law. It is the brightest cluster galaxy (BCG) in the CWAT-01 parent cluster. The surface brightness profile is very well fitted by the Sersic $r^{1 / n}$ law with $n=5, r_{\text {eff }}=8.2 \mathrm{kpc}$, and $\mu_{\text {eff }}=22.11 \mathrm{mag} \operatorname{arcsec}^{-2}$, consistent with values typical for brightest cluster galaxies (cD/D).

Applying several hydrodynamic models, taking ram pressure and buoyancy forces into account, to explain the observed bending of the radio jets of CWAT-01, the allowed range of the galaxy velocity relative to the ICM is approximately $300-550 \mathrm{~km} \mathrm{~s}^{-1}$. Both the upper and lower velocity are higher than is expected for dominant galaxies (i.e., BCGs) in relaxed systems.

The cluster hosting CWAT-01 (the CWAT-01 parent cluster) was detected in diffuse X-ray emission. The luminosity-weighted temperature of the cluster is $\sim 1.7 \mathrm{keV}$ consistent with poor cluster temperatures. The total mass within the $r_{500}$ radius is $\sim 5.8 \times$ $10^{13} M_{\odot}$. The cluster shows evidence for subclustering, both in diffuse X-ray emission and in the spatial distribution of galaxies found from the optical analysis applying the Voronoi tessellationbased approach (VTA). The distribution of early-type galaxies is not centrally concentrated; it is irregular and partitioned into two apparently distinct subclumps.

The CWAT-01 parent cluster itself is part of a larger cluster assembly consisting of a minimum of four clusters within $\sim 2 \mathrm{Mpc}$ distance identified via diffuse X-ray emission. The ICM temperatures of the three clusters surrounding the CWAT-01 parent cluster are in the $1.4-1.5 \mathrm{keV}$ range, consistent with temperatures of poor clusters. The total masses of the clusters within the $r_{500}$ radius are in the range of about $(4.1-4.4) \times 10^{13} M_{\odot}$.

The VTA results indicate that there is at least one more loose group that is likely bound to the system. From the X-ray detection limit for diffuse sources we infer that the total mass of this group must be less than $1.5 \times 10^{13} M_{\odot}$.

The whole cluster structure described in this paper is encompassed in a large-scale structure component, LSS 17, reported in Scoville et al. (2007b). LSS 17 is elongated in the north-south direction and extends $\sim 4 \mathrm{Mpc}$ along the major axis. Our results strongly indicate that we are witnessing the formation of a large cluster from an assembly of multiple clusters, consistent with the scenario of hierarchical structure formation. If this is the case, then the estimated minimum total mass of the final single cluster after the poor clusters merge would correspond to $M \sim 2.0 \times$ $10^{14} M_{\odot}$, or $\sim 20 \%$ of the Coma cluster mass. In this scenario, the large velocity of the CWAT-01 host galaxy relative to the ICM can easily be explained. The CWAT-01 parent cluster seems not 
to be relaxed; thus, a plausible explanation of the motion of the galaxy relative to the ICM is interaction of the two identified subclumps ( $\mathrm{SC} 1$ and $\mathrm{SC} 2$ ) within the cluster. On the other hand, we cannot rule out the gravitational influence of the other poor clusters as a cause for inducing such a velocity.

Resolving the detailed physics causing the bending of the radio jets of CWAT-01, the dynamical interplay between and within particular clusters, as well as the spectroscopic confirmation of the physical connection of the clusters, has to await the completion of the zCOSMOS program (Lilly et al. 2007). Nevertheless, our results support the idea that WAT galaxies are tracers of galaxy clusters, in particular dynamically young ones.

V. S. thanks Rachel Somerville and Klaus Meisenheimer for insightful discussions. C. C., E. S., and V. S. acknowledge support from NASA grant HST GO-09822.31-A; C. C. would like to acknowledge support from the Max Planck Society and the Alexander von Humboldt Foundation through the MaxPlanck-Forschungspreis 2005. A. F. acknowledges support from BMBF/DLR under grant 50 OR 0207, MPG and a partial support from NASA grant NNG04GF686. I. S. acknowledges the support of the European Community under a Marie Curie Intra-European Fellowship. C. S. B. acknowledges funding by the Deutsche Forschungsgemeinschaft. K. J. acknowledges support by the German DFG under grant SCHI 536/3-1.
The XMM-Newton project is an ESA Science Mission with instruments and contributions directly funded by ESA member states and the USA (NASA). The XMM-Newton project is supported by the Bundesministerium für Wirtschaft und Technologie/ Deutsches Zentrum für Luft- und Raumfahrt (BMWI/DLR, FKZ 50 OX 0001), the Max Planck Society, and the HeidenhainStiftung, and also by PPARC, CEA, CNES, and ASI. Part of this work was also supported by the Deutsches Zentrum für Luftund Raumfahrt, DLR project numbers 50 OR 0207 and 50 OR 0405 .

Funding for the Sloan Digital Sky Survey (SDSS) has been provided by the Alfred P. Sloan Foundation, the Participating Institutions, the National Aeronautics and Space Administration, the National Science Foundation, the US Department of Energy, the Japanese Monbukagakusho, and the Max Planck Society. The SDSS Web site is http://www.sdss.org.

The SDSS is managed by the Astrophysical Research Consortium (ARC) for the Participating Institutions. The Participating Institutions are the University of Chicago, Fermilab, the Institute for Advanced Study, the Japan Participation Group, the Johns Hopkins University, the Korean Scientist Group, Los Alamos Na2tional Laboratory, the Max Planck Institute for Astronomy, the Max Planck Institute for Astrophysics, New Mexico State University, the University of Pittsburgh, the University of Portsmouth, Princeton University, the US Naval Observatory, and the University of Washington.
Abazajian, K., et al. 2003, AJ, 126, 2081

Abazajian, K., et al. 2004, AJ, 128, 502 2005, AJ, 129, 1755

Adelman-McCarthy, J. K., et al. 2006, ApJS, 162, 38

Albert, C. E., White, R. A., \& Morgan, W. W. 1977, ApJ, 211, 309

Arnaud, M., Pointecouteau, E., \& Pratt, G. W. 2005, A\&A, 441, 893

Becker, R. H., White, R. L., \& Helfand, D. J. 1995, ApJ, 450, 559

Beers, T. C., \& Geller, M. J. 1983, ApJ, 274, 491

Beers, T. C., Kriessler, J. R., Bird, C. M., \& Huchra, J. P. 1995, AJ, 109, 874

Begelman, M. C., Rees, M. J., \& Blandford, R. D. 1979, Nature, 279, 770

Bird, C. M. 1994, AJ, 107, 1637

Blanton, E. L., Gregg, M. D., Helfand, D. J., Becker, R. H., \& Leighly, K. M. 2001, AJ, 121, 2915

Blanton, E. L., Gregg, M. D., Helfand, D. J., Becker, R. H., \& White, R. L. 2000, ApJ, 531, 118 2003, AJ, 125, 1635

Botzler, C. S., Snigula, J., Bender, R., Feulner, G., Goranova, Yu., \& Hopp, U. 2006, MNRAS, submitted

Brusa, M., et al. 2007, ApJS, 172, 353

Burns, J. O. 1981, MNRAS, 195, 523

Burns, J. O., Loken, C., Roettiger, K., Rizza, E., Bryan, G., Norman, M. L., Gómez, P., \& Owen, F. N. 2002, NewA Rev., 46, 135

Burns, J. O., Rhee, G., Owen, F. N., \& Pinkney, J. 1994, ApJ, 423, 94

Capak, P., et al. 2007, ApJS, 172, 99

Cavaliere, A., \& Fusco-Femiano, R. 1976, A\&A, 49, 137

Christiansen, W. A., Pacholczyk, A. G., \& Scott, J. S. 1981, ApJ, 251, 518

Coleman, G. D., Wu, C.-C., \& Weedman, D. W. 1980, ApJS, 43, 393

Condon, J. J., Cotton, W. D., Greisen, E. W., Yin, Q. F., Perley, R. A., Taylor,

G. B., \& Broderick, J. J. 1998, AJ, 115, 1693

Eilek, J. A. 1979, ApJ, 230, 373

Evrard, A. E. 1990, ApJ, 363, 349

Finoguenov, A., Arnaud, M., \& David, L. P. 2001a, ApJ, 555, 191

Finoguenov, A., Böhringer, H., \& Zhang, Y.-Y. 2005, A\&A, 442, 827

Finoguenov, A., Pietsch, W., Aschenbach, B., \& Miniati, F. 2004, A\&A, 415, 415

Finoguenov, A., Reiprich, T. H., \& Böhringer, H. 2001b, A\&A, 368, 749

Finoguenov, A., et al. 2007, ApJS, 172, 182

Frenk, C. S., Evrard, A. E., White, S. D. M., \& Summers, F. J. 1996, ApJ, 472, 460

Gomez, P. L., Ledlow, M. J., Burns, J. O., Pinkey, J., \& Hill, J. M. 1997, AJ, 114,1711

Graham, A., Lauer, T. R., Colless, M., \& Postman, M. 1996, ApJ, 465, 534

Gull, S. F., \& Northover, K. J. E. 1973, Nature, 244, 80

\section{REFERENCES}

Hardcastle, M. J., \& Sakelliou, I. 2004, MNRAS, 349, 560

Hasinger, G., et al. 2007, ApJS, 172, 29

Henry, J. P., Finoguenov, A., \& Briel, U. G. 2004, ApJ, 615, 181

Jaffe, W. J., \& Perola, G. C. 1973, A\&A, 26, 423

Jansen, F., et al. 2001, A\&A, 365, L1

Jing, Y. P., Borner, G., \& Valdarnini, R. 1995, MNRAS, 277, 630

Kim, R. S. J., et al. 2002, AJ, 123, 20

Kinney, A. L., Calzetti, D., Bohlin, R. C., McQuade, K., Storchi-Bergmann, T., \& Schmitt, H. R. 1996, ApJ, 467, 38

Koekemoer, M., et al. 2007, ApJS, 172, 196

Ledlow, M. J., Loken, C., Burns, J. O., Hill, J. M., \& White, R. A. 1996, AJ, 112,388

Lilly, S. J., et al. 2007, ApJS, 172, 70

Loken, C., Roettiger, K., Burns, J. O., \& Norman, M. 1995, ApJ, 445, 80

Lumb, D. H., Warwick, R. S., Page, M., \& De Luca, A. 2002, A\&A, 389, 93

Mahdavi, A., Finoguenov, A., Böhringer, H., Geller, M. J., \& Henry, J. P. 2005, ApJ, 622, 187

Malumuth, E. M., Kriss, G. A., Dixon, W. V. D., Ferguson, H. C., \& Ritchie, C. 1992, AJ, 104, 495

Markevitch, M. 1998, ApJ, 504, 27

Merritt, D. 1984, ApJ, 276, 26

Miley, G. 1980, ARA\&A, 18, 165

Mobasher, B., et al. 2007, ApJS, 172, 117

Morgan, W. W., Kayser, S., \& White, R. A. 1975, ApJ, 199, 545

Mulchaey, J. S. 2000, ARA\&A, 38, 289

Mulchaey, J. S., Davis, D. S., Mushotzky, R. F., \& Burstein, D. 2003, ApJS, 145,39

Novikov, D. I., Melott, A. L., Wilhite, B. C., Kaufman, M., Burns, J. O., Miller, C. J., \& Batuski, D. J. 1999, MNRAS, 304, L5

O’Dea, C. P., \& Owen, F. N. 1987, ApJ, 316, 95

O’Donoghue, A. A., Eilek, J. A., \& Owen, F. N. 1993, ApJ, 408, 428

Oegerle, W. R., \& Hill, J. M. 2001, AJ, 122, 2858

Osmond, J. P. F., \& Ponman, T. J. 2004, MNRAS, 350, 1511

Pacholczyk, A. G. 1970, Radio Astrophysics (San Francisco: Freeman)

Pinkney, J., Burns, J. O., \& Hill, J. M. 1994, AJ, 108, 2031

Quintana, H., \& Lawrie, D. G. 1982, AJ, 87, 1

Ramella, M., Boschin, W., Fadda, D., \& Nonino, M. 2001, A\&A, 368, 776

Ramella, M., Diaferio, A., Geller, M. J., \& Huchra, J. P. 1994, AJ, 107, 1623

Sakelliou, I., \& Merrifield, M. R. 2000, MNRAS, 311, 649

Sakelliou, I., Merrifield, M. R., \& McHardy, I. M. 1996, MNRAS, 283, 673

Schinnerer, et al. 2004, AJ, 128, 1974

. 2007, ApJS, 172, 46

Schombert, J. M. 1987, ApJS, 64, 643 
Scoville, N., et al. 2007a, ApJS, 172, 1 2007b, ApJS, 172, 150

Sersic, J. L. 1968, Atlas de Galaxias Australes (Cordoba: Observatorio Astronomico) Shewchuk, R. J. 1996, in Applied Computational Geometry: Towards Geometric Engineering, ed. M. C. Lin \& D. Manocha (Berlin: Springer), 203

Smith, R. K., Brickhouse, N. S., Liedahl, D. A., \& Raymond, J. C. 2001, ApJ, 556, L91

Springel, V., et al. 2005, Nature, 435, 629
Taniguchi, Y., et al. 2007, ApJS, 172, 9

Trump, J. R., et al. 2007, ApJS, 172, 383

van der Laan, H., \& Perola, G. C. 1969, A\&A, 3, 468

Vikhlinin, A., Markevitch, M., Murray, S. S., Jones, C., Forman, W., \& Van Speybroeck, L. 2005, ApJ, 628, 655

Watson, M. G., et al. 2001, A\&A, 365, L51

York, D. G., et al. 2000, AJ, 120, 1579 